\title{
An elementary construction of Anick's fibration
}

\author{
BRAYTON GRAY \\ STEPHEN THERIAULT
}

\begin{abstract}
Cohen, Moore, and Neisendorfer's work on the odd primary homotopy theory of spheres and Moore spaces, as well as the first author's work on the secondary suspension, predicted the existence of a $p$-local fibration $S^{2 n-1} \longrightarrow T_{2 n-1} \longrightarrow \Omega S^{2 n+1}$ whose connecting map is degree $p^{r}$. In a long and complex monograph, Anick constructed such a fibration for $p \geq 5$ and $r \geq 1$. Using new methods we give a much more conceptual construction which is also valid for $p=3$ and $r \geq 1$. We go on to establish an $H$ space structure on $T_{2 n-1}$ and use this to construct a secondary $E H P$ sequence for the Moore space spectrum.
\end{abstract}

\section{Introduction}

In $[6 ; 5 ; 15]$, Cohen, Moore and Neisendorfer proved a landmark result concerning the exponent of the homotopy groups of spheres localized at an odd prime $p$. When $p \geq 3$ and $r \geq 1$ they constructed a map $\pi_{n}: \Omega^{2} S^{2 n+1} \longrightarrow S^{2 n-1}$ such that the composition with the double suspension

$$
\Omega^{2} S^{2 n+1} \stackrel{\pi_{n}}{\longrightarrow} S^{2 n-1} \stackrel{E^{2}}{\longrightarrow} \Omega^{2} S^{2 n+1}
$$

is homotopic to the $p^{r}$-power map. The existence of such a map for $r=1$ was used to show that $p^{n}$ annihilates the $p$-torsion in $\pi_{*}\left(S^{2 n+1}\right)$.

In [4], Cohen, Moore and Neisendorfer raised the question of whether the map $\pi_{n}$ occurs in a fibration sequence ${ }^{1}$

$$
\Omega^{2} S^{2 n+1} \stackrel{\pi_{n}}{\longrightarrow} S^{2 n-1} \longrightarrow T_{2 n-1} \longrightarrow \Omega S^{2 n+1} .
$$

The first construction of such a fibration was accomplished for $p \geq 5$ by Anick [1] and was the subject of a 270 page book. There has been much interest in finding a simpler

\footnotetext{
${ }^{1}$ We will follow a convention suggested by Mahowald of indexing a family of infinite complexes by a subscript to denote the least dimension in which the reduced homology is nontrivial.
} 
construction. It is the purpose of this paper to give an elementary construction of the space $T_{2 n-1}$ and the fibration (A) which is valid for all odd primes.

The question of the existence of a fibration as in (A) appeared in another context at about the same time. In trying to understand the secondary suspension (see Cohen [3] and Mahowald [13]), the first author [9;10] was led to conjecture the existence of $(i-1)$-connected spaces $T_{i}$ which fit into secondary $E H P$ sequences

$$
\begin{gathered}
T_{2 n-1} \stackrel{E}{\longrightarrow} \Omega T_{2 n} \stackrel{H}{\longrightarrow} B W_{n} \\
T_{2 n} \stackrel{E^{\prime}}{\longrightarrow} \Omega T_{2 n+1} \stackrel{H^{\prime}}{\longrightarrow} B W_{n+1}
\end{gathered}
$$

where $B W_{n}$ is the classifying space of the fiber of the double suspension constructed by the first author in [8]. These EHP fibrations should fit together in such a way that the resulting spectrum $\left\{T_{i}\right\}$ is equivalent to the Moore spectrum $S^{0} \cup_{p^{r}} e^{1}$. The $T_{i}$ 's would then give a refinement of the secondary suspension into $2 p$ stages. The analysis indicated that $T_{2 n}$ is homotopy equivalent to $S^{2 n+1}\left\{p^{r}\right\}$, the fiber of the map of degree $p^{r}$ on $S^{2 n+1}$, and that $T_{2 n-1}$ would sit in the fibration sequence (A).

Our first objective is to construct a secondary Hopf invariant $H: \Omega T_{2 n} \longrightarrow B W_{n}$ for $p \geq 3$. This lets us define $T_{2 n-1}$ as the homotopy fiber of $H$. It follows easily that $T_{2 n-1}$ satisfies the fibration in (A) and the secondary EHP fibrations. We also show that the space we construct is homotopy equivalent to Anick's when $p \geq 5$.

The EHP viewpoint also predicted that the $T_{i}$ 's should have a rich structure. They should be homotopy associative and homotopy commutative $H$-spaces enjoying a certain universal property. Together, these properties would imply that the mod $-p^{r}$ homotopy classes of the $T_{i}$ 's could be represented by multiplicative maps. That is, letting $P^{i}\left(p^{r}\right)$ be the mod- $p^{r}$ Moore space of dimension $i$, there should be a one-to-one correspondence

$$
\left[P^{i+1}\left(p^{r}\right), T_{j}\right] \leftrightarrow\left\{\text { homotopy classes of } H \text {-maps from } T_{i} \text { to } T_{j}\right\} .
$$

The properties were easy to establish when $i$ is even [9]. Subsequent to Anick's work, Anick and the first author [2] constructed an $H$-space structure on $T_{2 n-1}$ by showing that, for each $n$, there is a $(2 n-1)$-connected co- $H$ space $G_{2 n}$ with the property that $T_{2 n-1}$ is a retract of $\Omega G_{2 n}$ and $G_{2 n}$ is a retract of $\Sigma T_{2 n-1}$. They also proved a semiuniversal property for $T_{2 n-1}$. This work depended heavily on the analysis of Anick in [1]. The other properties were later established by the second author [19].

Our second objective is to take advantage of our construction of the space $T_{2 n-1}$ to give a new, simpler construction of the space $G_{2 n}$ and prove all the properties in [2] for $p \geq 3$. Collectively, our results are as follows. 
Theorem 1.1 Suppose $p \geq 3$ and $r \geq 1$. Then the following hold:

(a) There is an $H$-fibration sequence

$$
\Omega^{2} S^{2 n+1} \stackrel{\pi_{n}}{\longrightarrow} S^{2 n-1} \longrightarrow T_{2 n-1} \longrightarrow \Omega S^{2 n+1}
$$

where the composition

$$
\Omega^{2} S^{2 n+1} \stackrel{\pi_{n}}{\longrightarrow} S^{2 n-1} \stackrel{E^{2}}{\longrightarrow} \Omega^{2} S^{2 n+1}
$$

is the $p^{r}$-power map.

(b) There is a fibration sequence

$$
\Omega G \stackrel{h}{\longrightarrow} T_{2 n-1} \longrightarrow R \longrightarrow G_{2 n}
$$

where $h$ has a right homotopy inverse $g: T_{2 n-1} \longrightarrow \Omega G_{2 n}$ so that

$$
\Omega G_{2 n} \simeq T_{2 n-1} \times \Omega R
$$

with $R$ a wedge of mod- $p^{s}$ Moore spaces for $s \geq r$.

(c) The adjoint of $g$,

$$
\tilde{g}: \Sigma T_{2 n-1} \longrightarrow G_{2 n},
$$

has a right homotopy inverse $f: G_{2 n} \longrightarrow \Sigma T_{2 n-1}$ and there is a homotopy equivalence

$$
\Sigma T_{2 n-1} \simeq G_{2 n} \vee W
$$

where $W$ is a wedge of mod- $p^{s}$ Moore spaces for $s \geq r$.

(d) There are secondary EHP fibrations

$$
\begin{gathered}
W_{n} \stackrel{P}{\longrightarrow} T_{2 n-1} \stackrel{E}{\longrightarrow} \Omega T_{2 n} \stackrel{H}{\longrightarrow} B W_{n} \\
W_{n+1} \stackrel{P^{\prime}}{\longrightarrow} T_{2 n} \stackrel{E^{\prime}}{\longrightarrow} \Omega T_{2 n+1} \stackrel{H^{\prime}}{\longrightarrow} B W_{n+1}
\end{gathered}
$$

where $T_{2 n}=S^{2 n+1}\left\{p^{r}\right\}$, and there is an equivalence of spectra

$$
\left\{T_{i}\right\} \simeq S^{0} \cup_{p^{r}} e^{1} .
$$

Our methods are simpler and more direct than those of Anick. He constructed $T_{2 n-1}$ as a retract of a loop space $\Omega D$, where $D$ is an infinite dimensional CW-complex whose bottom two cells are the mod $-p^{r}$ Moore space $P^{2 n+1}\left(p^{r}\right)$ and whose other cells come from iteratively attaching certain Moore spaces in a delicately prescribed fashion. A great deal of his effort was directed towards constructing the attaching maps, and this necessitated the introduction of many new techniques. The restriction 
to primes strictly larger than 3 was due to a heavy reliance on differential graded Lie algebras which require that the primes 2 and 3 be inverted in order for the Lie identities to be satisfied. By contrast, we construct the space $T_{2 n-1}$ directly for all $p \geq 3$ without reference to the space $D$ and without reference to differential graded Lie algebras. Section 2 is devoted to Extension Theorem 2.2 which introduces a new technique for doing obstruction theory in principal fibrations. This is the main tool for all our results. In Section 3 we construct the fibration in Theorem 1.1 (a), without the $H$-space structure. In Section 4 we use the extension theorem again in an elaborate induction to obtain the spaces $G_{2 n}$ and the $H$ space structure on $T_{2 n-1}$. Throughout the induction we reproduce some of the delicate properties of $G_{2 n}$ and $T_{2 n-1}$ which first appeared in [2].

The new methods may be useful in positively resolving a long-standing conjecture that the fiber $W_{n}$ of the double suspension is a double loop space at odd primes. Including dimension and torsion parameters, the space $T_{2 n p-1}(p)$ gives a candidate for a double delooping: potentially $W_{n} \simeq \Omega^{2} T_{2 n p-1}(p)$. Such a homotopy equivalence would have deep implications in homotopy theory, one of which being a much better understanding of the differentials in the EHP spectral sequence calculating the homotopy groups of spheres.

This paper is the result of combining separate efforts by the two authors. The second author discovered the extension theorem and obtained part (a) of Theorem 1.1 without the $H$-space structure, as well as part (d). The first author later found a simpler application of the extension theorem to obtain the map $H$ and Corollary 3.5, Theorem 3.6, Proposition 3.7, Theorem 3.8 and Corollary 3.9 below, as well as a further application of the extension theorem to obtain parts (b) and (c), and the $H$-space structure.

The authors would like to warmly thank the referee for a sustained effort in carefully reading the paper and its revisions, and for making many helpful comments and suggestions.

\section{The extension theorem}

In this section we establish an extension theorem for principal fibrations defined over mapping cones. Let

$$
E \stackrel{\pi}{\longrightarrow} X \cup_{\theta} C A
$$


be a principal fibration classified by a map $\varphi: X \cup_{\theta} C A \longrightarrow Y$. We compare this to the induced fibration over $X$ :

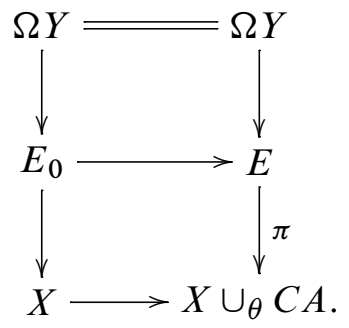

In Extension Theorem 2.2 we will give conditions for when a map $E_{0} \longrightarrow B$ extends to a map $E \longrightarrow B$. To motivate the underlying idea, observe that there is a pushout

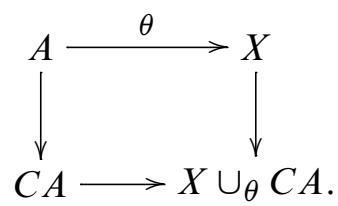

Map all four corners of this square into $Y$ by composing with the map $\varphi: X \cup_{\theta} C A \longrightarrow$ $Y$. Appropriately turning maps into fibrations, we obtain fibration sequences $E \longrightarrow$ $X \cup_{\theta} C A \longrightarrow Y, E_{0} \longrightarrow X \longrightarrow Y, C A \times \Omega Y \longrightarrow C A \longrightarrow Y$, and $A \times \Omega Y \longrightarrow A \longrightarrow Y$. By [8] or Mather's Cube Lemma [14], these fibres fit into a homotopy pushout

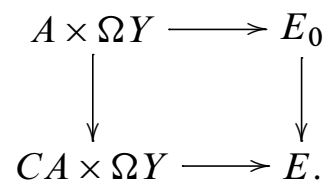

We wish to produce an extension of $f_{0}: E_{0} \longrightarrow B$ as a pushout map $f: E \longrightarrow B$. To obtain this, we need to produce a map $C A \times \Omega Y \longrightarrow B$ and show that both it and $f_{0}$ are homotopic when restricted to $A \times \Omega Y$. This leads to two issues. One is to identify the maps which appear in the homotopy pushout (1). We do this by constructing the homotopy pushout for $E$ from first principles. The other issue is to impose conditions on the spaces and maps in (1) and on the space $B$ which will guarantee the existence of an extension. We will impose three conditions: $A$ is a co- $H$ space, $B$ is a connected $H$-space whose $q$-power map is null homotopic, and the restriction of $A \times \Omega Y$ to $E_{0}$ is divisible by $q$. It may be worth noting that the exponent condition on $B$ will be played off of the divisibility condition for the map $A \longrightarrow E_{0}$.

To set things up, observe as in (1) that the map $A \longrightarrow X$ lifts to $E_{0}$. There may be many inequivalent choices of such a lift. By the homotopy lifting property, we can 
extend this lift to a map of pairs $\phi:(C A, A) \longrightarrow\left(E, E_{0}\right)$ such that the composite

$$
(C A, A) \stackrel{\phi}{\longrightarrow}\left(E, E_{0}\right) \stackrel{\pi}{\longrightarrow}\left(X \cup_{\theta} C A, X\right)
$$

induces a cohomology isomorphism. Again, note that there may be many inequivalent choices of $\phi$ with this property. In what follows, all spaces will have the homotopy type of $p$-local CW-complexes with $p>2$.

Extension Theorem 2.2 Let $A$ be a co- $H$ space and $B$ be a connected $H$-space whose $q$-power map is null homotopic. Let $\phi:(C A, A) \rightarrow\left(E, E_{0}\right)$ be a map such that the composition

$$
(C A, A) \stackrel{\phi}{\longrightarrow}\left(E, E_{0}\right) \stackrel{\pi}{\longrightarrow}\left(X \cup_{\theta} C A, X\right)
$$

induces a cohomology isomorphism. Suppose that the restriction $\left.\phi\right|_{A}: A \longrightarrow E_{0}$ is divisible by $q$ in the co- $H$ structure on $A$. Then the restriction

$$
[E, B] \longrightarrow\left[E_{0}, B\right]
$$

is onto. Consequently, any map $E_{0} \longrightarrow B$ extends to a map $E \longrightarrow B$.

Proof Let $s:(C A, A) \longrightarrow\left(X \cup_{\theta} C A, X\right)$ be the standard map defining the mapping cone. Observe that $\pi \phi \neq s$, in general. We first modify $\phi$ so that this does happen. We do this by constructing a pullback diagram

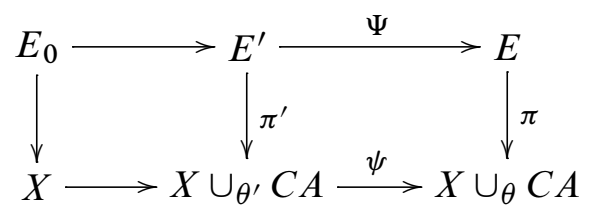

and a map $\phi^{\prime}:(C A, A) \longrightarrow\left(E^{\prime}, E_{0}\right)$ such that

(a) $\left.\phi^{\prime}\right|_{A}=\left.\phi\right|_{A}$;

(b) $\pi^{\prime} \phi^{\prime}=s^{\prime}:(C A, A) \longrightarrow\left(X \cup_{\theta^{\prime}} C A, X\right)$, the standard map;

(c) $\Psi: E^{\prime} \longrightarrow E$ is a homotopy equivalence.

Let $\theta^{\prime}: A \longrightarrow X$ be the restriction of $\pi \phi$ to $A$. Define $\psi: X \cup_{\theta^{\prime}} C A \longrightarrow X \cup_{\theta} C A$ extending the identity on $X$ with the map $\pi \phi: C A \longrightarrow X \cup_{\theta} C A$. Define $E^{\prime}$ as the pullback of $\pi$ and $\psi$. By the pullback property of $E^{\prime}$, we can define $\phi^{\prime}$ such that $\Psi \phi^{\prime}=$ $\phi$ and $\pi^{\prime} \phi^{\prime}=s^{\prime}$. Then $\psi s^{\prime}=\gamma \pi^{\prime} \phi^{\prime}=\pi \Psi \phi^{\prime}=\pi \phi$. Since $\pi \phi$ induces an isomorphism in cohomology and $s^{\prime}$ is an excision, $\psi:\left(X \cup_{\theta^{\prime}} C A, X\right) \longrightarrow\left(X \cup_{\theta} C A, X\right)$ induces a 
cohomology isomorphism as well. Hence $\psi$ is a homotopy equivalence and it follows that $\Psi$ is a homotopy equivalence.

We are therefore reduced to considering the case when $\pi \phi=s$. Since both fibrations are principal fibrations, there is an action of $\Omega Y$ on the total space

$$
\Omega Y \times E \longrightarrow E
$$

which restricts to an action on $E_{0}$

$$
\Omega Y \times E_{0} \longrightarrow E_{0} .
$$

Consider the composition

$$
\gamma:(\Omega Y \times C A, \Omega Y \times A) \stackrel{1 \times \phi}{\longrightarrow}\left(\Omega Y \times E, \Omega Y \times E_{0}\right) \longrightarrow\left(E, E_{0}\right) .
$$

Identifying $\Omega Y \times A \subset \Omega Y \times C A$ with its image under $\gamma$ in $E_{0}$, define $\bar{E}=E_{0} \cup$ $\Omega Y \times C A$ by the pushout

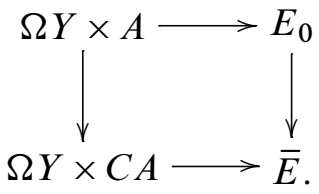

From the map $\gamma$ we obtain a pushout map

$$
\Gamma: \bar{E}=E_{0} \cup \Omega Y \times C A \longrightarrow E .
$$

Observe that there is a homotopy commutative square

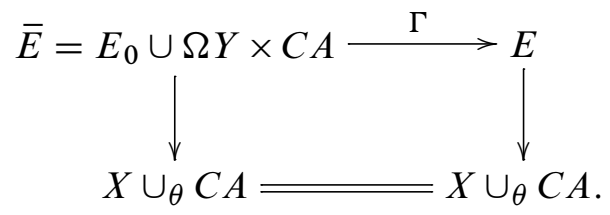

By [8], the left hand vertical map is a quasi-fibration with quasi-fiber $\Omega Y$. Since quasi-fibrations induce long exact sequences in homotopy, the 5-lemma implies that $\Gamma$ is a homotopy equivalence.

Let $f_{0}: E_{0} \longrightarrow B$. We will construct an extension $\bar{f}: \bar{E} \longrightarrow B$ and hence an extension $f: E \longrightarrow B$ via the homotopy equivalence $\Gamma$. Since $\bar{E}$ is a homotopy pushout, it suffices to construct a map

$$
g: \Omega Y \times C A \longrightarrow B
$$

such that the composite

$$
\Omega Y \times A \stackrel{\gamma}{\longrightarrow} E_{0} \stackrel{f_{0}}{\longrightarrow} B
$$


is homotopic to the composite

$$
\Omega Y \times A \longrightarrow \Omega Y \times C A \stackrel{g}{\longrightarrow} B .
$$

Define $g$ as the composition

$$
\Omega Y \times C A \stackrel{\pi_{1}}{\longrightarrow} \Omega Y \stackrel{\iota}{\longrightarrow} E_{0} \stackrel{f_{0}}{\longrightarrow} B
$$

where $\pi_{1}$ is the projection and $\iota$ is the map in the given principal fibration $\Omega Y \longrightarrow$ $E_{0} \longrightarrow X$. Then we are reduced to showing that the compositions

$$
\begin{aligned}
& \alpha: \Omega Y \times A \stackrel{\gamma}{\longrightarrow} E_{0} \stackrel{f_{0}}{\longrightarrow} B \\
& \beta: \Omega Y \times A \stackrel{\pi_{1}}{\longrightarrow} \Omega Y \stackrel{\iota}{\longrightarrow} E_{0} \stackrel{f_{0}}{\longrightarrow} B
\end{aligned}
$$

are homotopic. In Lemma 2.4, we will show in general that if $\alpha, \beta: X \times A \longrightarrow B$ are two maps with $\left.\left.\alpha\right|_{X \times *} \sim \beta\right|_{X \times *}$, then $\alpha \circ(1 \times q) \sim \beta \circ(1 \times q)$. Assuming this for the moment, apply the lemma in our case. By definition, $\gamma: \Omega Y \times A \longrightarrow E_{0}$ is the composition

$$
\Omega Y \times A \stackrel{1 \times \phi}{\longrightarrow} \Omega Y \times E_{0} \longrightarrow E_{0},
$$

and by hypothesis $\left.\phi\right|_{A}: A \longrightarrow E_{0}$ is divisible by $q$. Let $\left.\phi\right|_{A}=q \cdot \phi^{\prime}$. Consequently, $\gamma=\gamma^{\prime} \circ(1 \times q)$ (where $\gamma^{\prime}$ is constructed by replacing $\phi$ with $\left.\phi^{\prime}\right)$ and $\alpha=\alpha^{\prime} \circ(1 \times q)$ where $\alpha^{\prime}=f_{0} \circ \gamma^{\prime}$. Also, the projection in the definition of $\beta$ implies that $\beta=\beta \circ(1 \times q)$. Thus by Lemma 2.4, $\alpha^{\prime} \circ(1 \times q)$ is homotopic to $\beta$. That is, $\alpha$ is homotopic to $\beta$, as required.

It remain to prove Lemma 2.4. This will rely on Lemma 2.3, which summarizes some well known properties of connected $H$ spaces.

Lemma 2.3 Let $B$ be a connected $H$-space and suppose $u$, $v$ are maps $Z \longrightarrow B$. Then there is a difference map $\delta(u, v): Z \longrightarrow B$ such that

(a) $\delta(u, v) \sim *$ if and only if $u \sim v$;

(b) if $h: W \longrightarrow Z$, then $\delta(u \circ h, v \circ h) \sim \delta(u, v) \circ h$;

(c) if $C \subset Z$ and $\left.u\right|_{C}=\left.v\right|_{C}$, then $\left.\delta(u, v)\right|_{C} \sim *$.

Proof Write $x, y: B \times B \longrightarrow B$ for the projections onto the first and second coordinates. Then there is a homotopy equivalence $e: B \times B \longrightarrow B \times B$ given by 
$e=(x, \mu(x, y))$. Let $\delta: B \times B \longrightarrow B$ be the second coordinate of a homotopy inverse for $e$. Then

$$
\begin{aligned}
& \delta(x, \mu(x, y)) \sim y \\
& \mu(x, \delta(x, y)) \sim y .
\end{aligned}
$$

Define $\delta(u, v)$ as the composition

$$
Z \stackrel{u \times v}{\longrightarrow} B \times B \stackrel{\delta}{\longrightarrow} B .
$$

If $\delta(u, v) \sim *$, then $u \sim \mu(u, *) \sim \mu(u, \delta(u, v)) \sim v$ by (2.3.2). On the other hand, if $u \sim v$, then $\delta(u, v) \sim \delta(u, u) \sim \delta(u, \mu(u, *)) \sim *$ by (2.3.1). This proves part (a). Part (b) follows by naturality. For part (c), apply (b) with $W=C$ and $h$ the inclusion. It follow that $\left.\delta(u, v)\right|_{C} \sim *$.

Lemma 2.4 Let $B$ be a connected $H$-space whose $q$-power map is null homotopic. Suppose $\alpha, \beta: X \times A \longrightarrow B$ are two maps with $\left.\left.\alpha\right|_{X \times *} \sim \beta\right|_{X \times *}$. Then $\alpha \circ(1 \times q) \sim$ $\beta \circ(1 \times q)$.

Proof Write $\delta=\delta(\alpha, \beta): X \times A \longrightarrow B$ for the difference element defined in Lemma 2.3. Since $\left.\delta\right|_{X \times *}$ is null homotopic, we obtain a homotopy commutative diagram

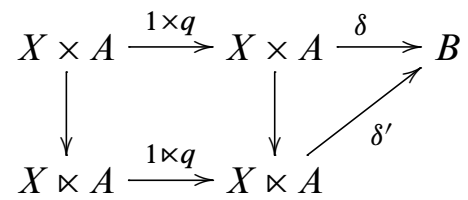

for some map $\delta^{\prime}$. Since $X \ltimes\left(A_{1} \vee A_{2}\right)$ is homeomorphic to $\left(X \ltimes A_{1}\right) \vee\left(X \ltimes A_{2}\right)$, the co- $H$ structure on $A$ induces a co- $H$ structure on $X \ltimes A$ and $1 \ltimes q$ is the degree $q$ self-map on $X \ltimes A$. The co- $H$ space structure on $X \ltimes A$ induces the same group structure on $[X \ltimes A, B]$ as the $H$-space structure on $B$. Thus the composite

$$
X \ltimes A \stackrel{1 \ltimes q}{\longrightarrow} X \ltimes A \stackrel{\delta^{\prime}}{\longrightarrow} B
$$

is homotopic to the composite

$$
X \ltimes A \stackrel{\delta^{\prime}}{\longrightarrow} B \stackrel{q}{\longrightarrow} B .
$$

By assumption, the $q$-power map on $B$ is null homotopic. Thus $q \circ \delta^{\prime}$ is null homotopic and so $\delta^{\prime} \circ(1 \ltimes q)$ is null homotopic. Therefore $\delta \circ(1 \times q)=\delta(\alpha, \beta) \circ(1 \times q)$ is null homotopic. On the other hand, by Lemma 2.3 (b), $\delta(\alpha, \beta) \circ(1 \times q) \sim \delta(\alpha \circ(1 \times q)$, $\beta \circ(1 \times q))$, implying that the right hand side is null homotopic. Hence Lemma 2.3 (a) implies that $\alpha \circ(1 \times a) \sim \beta \circ(1 \times q)$. 


\section{The construction of the space $T_{2 n-1}$}

The purpose of this section is to construct the spaces $T_{2 n-1}$ and produce several fibration sequences. We begin our discussion with the Moore space

$$
P^{k}\left(p^{r}\right)=S^{k-1} \cup p^{r} e^{k}
$$

which we will abbreviate as $P^{k}$. Let us fix some notation by defining a diagram of fibration sequences induced by the lower right hand corner

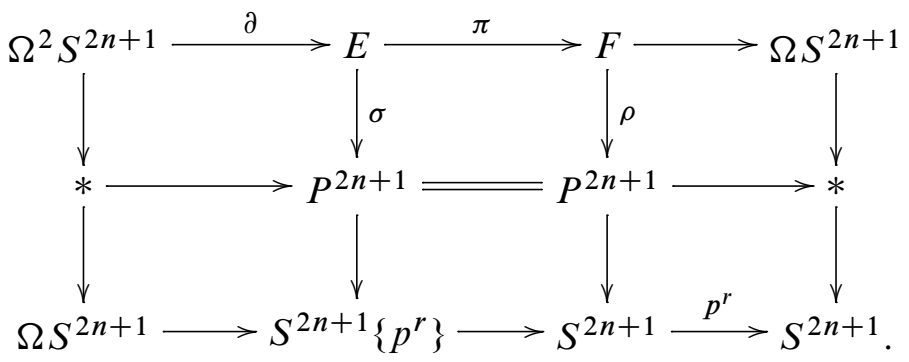

The spaces $E$ and $F$ were first introduced in $[5 ; 6]$. It is easy to see that

$$
H^{i}(F)= \begin{cases}\mathbb{Z} & i=2 k n, \\ 0 & i \neq 2 k n\end{cases}
$$

from the cohomology Serre spectral sequence for the fibration

$$
\Omega S^{2 n+1} \stackrel{\partial}{\longrightarrow} F \stackrel{\rho}{\longrightarrow} P^{2 n+1}
$$

which is induced from the path space fibration over $S^{2 n+1}$.

In [5], Cohen, Moore and Neisendorfer introduced certain iterated relative Samelson products $x_{i}: P^{2 n i-1} \longrightarrow \Omega F$. We will work with their adjoints $\hat{x}_{i}: P^{2 n i} \longrightarrow F$ which can be thought of as iterated relative Whitehead products. The following lemma was certainly known by them.

Lemma 3.2 For $i \geq 1$, there are maps $\hat{x}_{i}: P^{2 n i} \longrightarrow F$ such that

(a) if $Z$ is an $H$ space and $f: P^{2 n+1} \rightarrow Z$, then $f \rho \hat{x}_{i} \sim *$ for $i>1$;

(b) $\hat{x}_{i}: P^{2 n i} \longrightarrow F$ induces an epimorphism in integral cohomology.

Proof Let $\hat{x}_{1}: P^{2 n} \longrightarrow F$ be a lift of the standard map $P^{2 n} \longrightarrow S^{2 n} \longrightarrow P^{2 n+1}$. Since $\partial: \Omega S^{2 n+1} \longrightarrow F$ has degree $p^{n}$ in cohomology, $\hat{x}_{1}$ is unique up to homotopy 
and (b) clearly holds. Having constructed $\hat{x}_{i}$, consider the map of fibrations

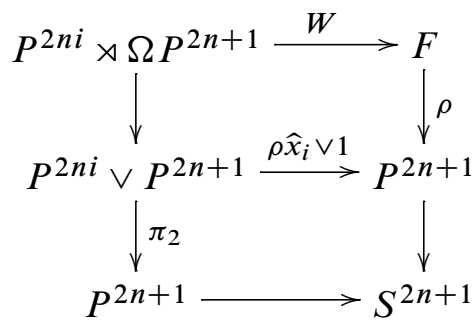

where the fibration on the left is the universal fibration for relative Whitehead products and the induced map of fibres defines $W$. Let $\hat{x}_{i+1}$ be the composition

$$
P^{2 n(i+1)} \longrightarrow P^{2 n i} \rtimes P^{2 n} \longrightarrow P^{2 n i} \rtimes \Omega P^{2 n+1} \stackrel{W}{\longrightarrow} F
$$

where the first map has a left homotopy inverse and projects trivially to $P^{2 n i}$ and the second map is the natural inclusion. To prove (a), suppose that $f: P^{2 n+1} \rightarrow Z$ where $Z$ is an $H$ space. Then we can construct a homotopy commutative diagram:

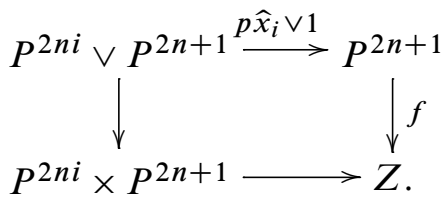

Now since $f \hat{x}_{i+1}$ factors through $P^{2 n i} \times P^{2 n+1}$, it is null homotopic by construction. To prove (b) apply induction and compare the Serre spectral sequences for the two fibrations.

Since $S^{2 n+1}\left\{p^{r}\right\}$ is an $H$ space, the classes $\rho \hat{x}_{i}$ lift to classes $y_{i}: P^{2 n i} \longrightarrow E$ for $i>1$. Thus $\sigma y_{i} \sim \rho \hat{x}_{i}$ and $\rho \pi=\sigma$ imply that $\rho\left(\pi y_{i}-\hat{x}_{i}\right)$ is null homotopic. Consequently $\pi y_{i}-\hat{x}_{i}=\partial u_{i}$ for some maps $u_{i}: P^{2 n i} \longrightarrow \Omega S^{2 n+1}$. Since $\partial^{*}: H^{2 n i}(F) \longrightarrow H^{2 n i}\left(\Omega S^{2 n+1}\right)$ has degree $p^{r},\left(\pi y_{i}-\hat{x}_{i}\right)^{*}=0$ and we obtain the following.

Lemma 3.3 For $i>1$, the composite $H^{2 n i}(F) \stackrel{\pi^{*}}{\longrightarrow} H^{2 n i}(E) \stackrel{y_{i}^{*}}{\longrightarrow} H^{2 n i}\left(P^{2 n i}\right)$ is an epimorphism.

Define $F_{(i)}$ as the $2 n i$ skeleton of $F$. Define $E_{(i)}$ by the homotopy pullback

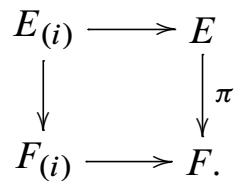


Note that by (3.1) the fibre of $\pi$ is $\Omega^{2} S^{2 n+1}$, and so the same is true of the induced map $E_{(i)} \longrightarrow F_{(i)}$. Including $F_{(i-1)}$ as the $(2 n(i-1))$-skeleton of $F_{(i)}$, we obtain a homotopy pullback diagram

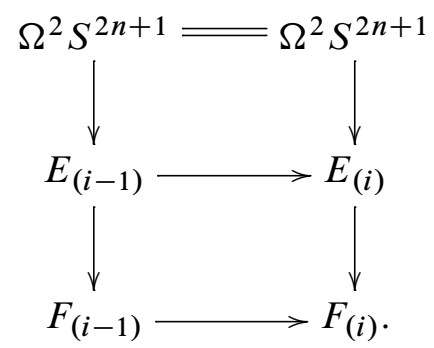

Let $\mathcal{X}_{i}:\left(D^{2 n i}, S^{2 n i-1}\right) \longrightarrow\left(P^{2 n i}, S^{2 n i-1}\right)$ be the characteristic map of the $2 n i$ cell. Then $\left.\mathcal{X}_{i}\right|_{S^{2 n i-1}}$ has degree $p^{r}$. The composition

$$
\theta:\left(D^{2 n i}, S^{2 n i-1}\right) \stackrel{\mathcal{X}_{i}}{\longrightarrow}\left(P^{2 n i}, S^{2 n i-1}\right) \stackrel{y_{i}}{\longrightarrow}\left(E_{(i)}, E_{(i-1)}\right) \stackrel{\pi}{\longrightarrow}\left(F_{(i)}, F_{(i-1)}\right)
$$

induces a cohomology isomorphism by Lemma 3.3. Thus there is an equivalence

$$
F_{(i)}=F_{(i-1)} \cup_{\theta} e^{2 n i} .
$$

The restriction of $\theta$ to $S^{2 n i-1}$ is divisible by $p^{r}$ so the conditions of Extension Theorem 2.2 are satisfied with $q=p^{r}$. Therefore we have proved the following.

Theorem 3.4 If $B$ is a connected $H$ space whose $p^{r}$-power map is null homotopic, then for $i>1$ any map $E_{(i-1)} \longrightarrow B$ extends to a map $E_{(i)} \longrightarrow B$.

In [8], a classifying space $B W_{n}$ of the fiber of the double suspension was constructed, along with a fibration sequence

$$
S^{2 n-1} \stackrel{E^{2}}{\longrightarrow} \Omega^{2} S^{2 n+1} \stackrel{v}{\longrightarrow} B W_{n} .
$$

Corollary 3.5 There is a map $v^{E}: E \longrightarrow B W_{n}$ such that the composition

$$
\Omega^{2} S^{2 n+1} \stackrel{\partial}{\longrightarrow} E \stackrel{\nu^{E}}{\longrightarrow} B W_{n}
$$

is homotopic to $v$.

Proof Since $F_{(1)}=S^{2 n}$, we have the fibration

$$
\Omega^{2} S^{2 n+1} \longrightarrow E_{(1)} \longrightarrow S^{2 n} \longrightarrow \Omega S^{2 n+1} .
$$


This fibration was analyzed in [8] and it was shown that $E_{(1)} \simeq S^{4 n-1} \times B W_{n}$ in such a way that the composition

$$
\Omega^{2} S^{2 n+1} \stackrel{\partial}{\longrightarrow} S^{4 n-1} \times B W_{n} \stackrel{\pi_{2}}{\longrightarrow} B W_{n}
$$

is homotopic to $v$. It was also shown that for $p \geq 5 B W_{n}$ is a homotopy associative $H$ space. The $H$ space structure on $B W_{n}$ was shown to be homotopy associative for $p=3$ and that the $p$-power map on $B W_{n}$ is null homotopic in [20]. Thus for $i>1$ we can apply Theorem 3.4 to construct maps $v_{i}: E_{(i)} \longrightarrow B W_{n}$ by induction such that $\nu_{i} \partial_{i} \sim v$. Since $E=\bigcup E_{(i)}$, we define $v^{E}: E \longrightarrow B W_{n}$ by $v^{E} \mid E_{i}=v_{i}$.

Theorem 3.6 There is a diagram of fibrations

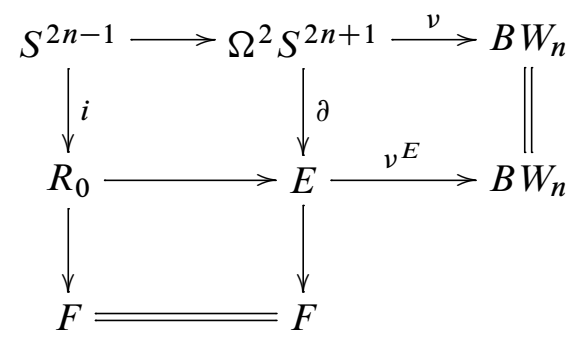

with $i$ null homotopic and so $\Omega F \simeq S^{2 n-1} \times \Omega R_{0}$.

Proof The space $R_{0}$ is defined as the fiber of $v^{E}$. Since the fibration

$$
\Omega^{2} S^{2 n+1} \stackrel{\partial}{\longrightarrow} E \longrightarrow F
$$

is induced by a map to $\Omega S^{2 n+1}$ which induces an isomorphism in $H_{2 n}()$, the map $\Omega F \rightarrow S^{2 n-1}$ induces an isomorphism in $H_{2 n-1}()$ and hence has a right homotopy inverse.

It is worth noting at this point that the space $\Omega F_{0}$ is split in [6]; consequently there is a homotopy decomposition

$$
\Omega R_{0} \simeq \prod_{i \geq 1} S^{2 n p^{i}-1}\left\{p^{r+1}\right\} \times \Omega P(n, r)
$$

where $P(n, r)$ is a complicated wedge of $\bmod -p^{r}$ Moore spaces. The fact that the product on the right is a loop space and is mapped to $\Omega F$ by a loop map is not obvious from their analysis. The cohomology structure of $R_{0}$ is rather simple. 
Proposition 3.7 We have

$$
H^{m}\left(R_{0}\right)= \begin{cases}\mathbb{Z} / p^{r} i \mathbb{Z} & \text { if } m=2 n i \text { and } i>1, \\ 0 & \text { otherwise. }\end{cases}
$$

Moreover, there is a choice of generators $e_{i} \in H^{2 m i}\left(R_{0}\right)$ such that $e_{i} e_{j}=p^{r}\left(\begin{array}{c}i+j \\ i\end{array}\right) e_{i+j}$ when $i, j>0$.

Proof Apply the Serre spectral sequence to the fibration $S^{2 n-1} \longrightarrow R_{0} \longrightarrow F$ in Theorem 3.6.

We now construct the space $T$ in Theorem 1.1 and prove the existence of the fibrations in parts (a) and (d), leaving the $H$-structure to the next section. By Diagram (3.1) there is a fibration sequence

$$
\Omega S^{2 n+1}\left\{p^{r}\right\} \stackrel{\tau}{\longrightarrow} E \stackrel{\sigma}{\longrightarrow} P^{2 n+1} \longrightarrow S^{2 n+1}\left\{p^{r}\right\} .
$$

Define $H$ by the composition

$$
H: \Omega S^{2 n+1}\left\{p^{r}\right\} \stackrel{\tau}{\longrightarrow} E \stackrel{\nu^{E}}{\longrightarrow} B W_{n} .
$$

Note that $H$ can be regarded as a secondary Hopf invariant. Define $T$ as the homotopy fiber of $H$. Then Theorem 3.6 implies the following.

Theorem 3.8 There is a diagram of fibrations

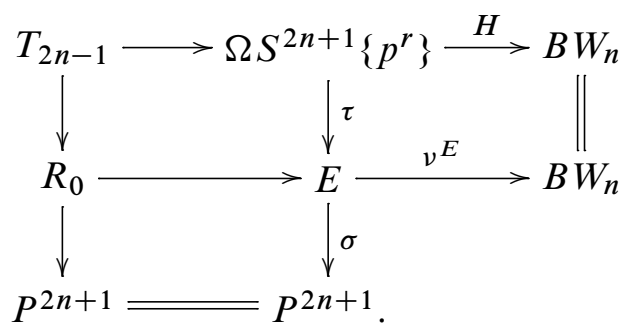

The connecting maps for the vertical fibrations in Theorem 3.8 immediately give the following.

Corollary 3.9 There is a homotopy commutative diagram

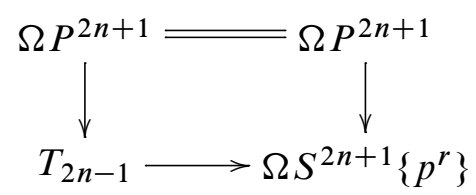

where the right map is the loop of the inclusion of the bottom Moore space. 
Continuing the diagram in (3.1), we have

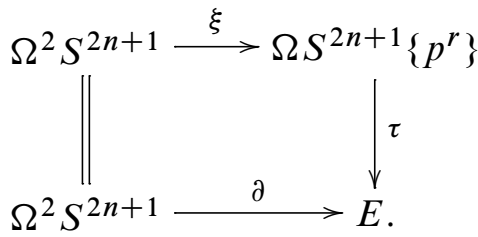

Applying Theorems 3.6 and 3.8 gives

$$
H \xi \sim v^{E} \tau \xi \sim v^{E} \partial \sim v,
$$

and we conclude:

Theorem 3.10 There is a diagram of fibrations

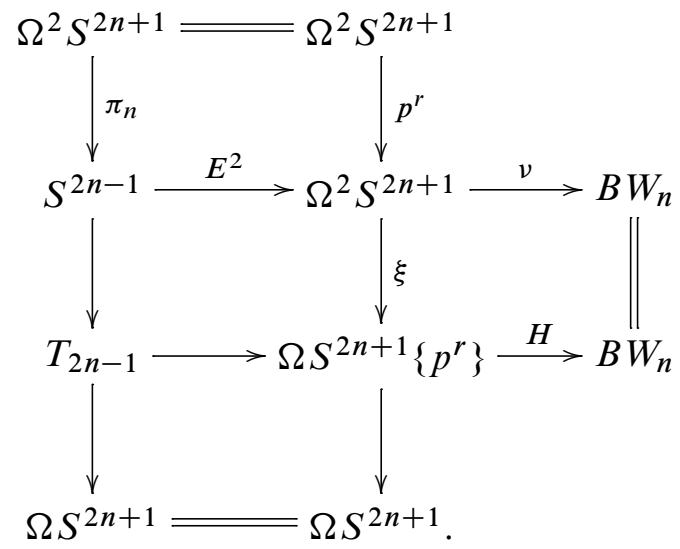

In particular, the top square in Theorem 3.10 is Cohen, Moore, and Neisendorfer's factorization of the $p^{r}$-power map on $\Omega^{2} S^{2 n+1}$. Since $\pi_{n}$ has degree $p^{r}$, we have the following corollary.

Corollary 3.11 There is a homotopy commutative diagram

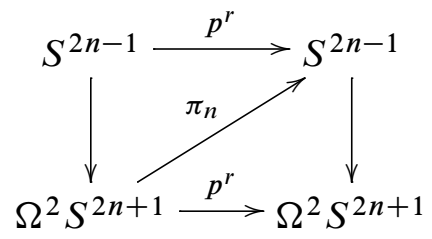

for each $r \geq 1$. 


\section{The construction of $G_{2 n}$ and the $H$-space structure on $T_{2 n-1}$}

In this section we will fix $n$ and abbreviate $T_{2 n-1}$ and $G_{2 n}$ as $T$ and $G$. We will need to filter $T$ by skeleta and write $T^{m}$ for the $m$ skeleton.

Our purpose is to construct an $H$-space structure on $T$. In fact we do more than that. We construct a corresponding co- $H$ space $G$ in the sense of [11]; ie, we construct a $(2 n-2)-$ connected space $G$ and maps

$$
\begin{aligned}
& f: G \longrightarrow \Sigma T \\
& g: T \longrightarrow \Omega G \\
& h: \Omega G \longrightarrow T
\end{aligned}
$$

such that the compositions

$$
\begin{aligned}
& G \stackrel{f}{\longrightarrow} \Sigma T \stackrel{\text { g}}{\longrightarrow} G \\
& T \stackrel{g}{\longrightarrow} \Omega G \stackrel{h}{\longrightarrow} T
\end{aligned}
$$

are homotopic to the identity, where $\widetilde{g}$ is the adjoint of $g$. We go on to derive several interesting results from this structure.

We will write $T^{m}$ for the $m$-skeleton of $T$. We will also reintroduce the torsion parameter for Moore spaces as we will need to consider mod- $p^{s}$ Moore spaces $P^{m}\left(p^{s}\right)$ for $s \neq r$. The space $G$ will be filtered by the $2 n p^{k}+1$ skeleton which we will abbreviate as $G_{k}$. These will be constructed inductively starting with $G_{-1}=*$. We will construct a map

$$
\alpha_{k}: P^{2 n p^{k}}\left(p^{r+k}\right) \longrightarrow G_{k-1}
$$

and define $G_{k}$ as the mapping cone of $\alpha_{k}$.

One of the features of [11] is that the spaces $G$ and $T$ come with a fibration

$$
T \longrightarrow R \longrightarrow G .
$$

Thus we seek to construct $G$ inductively over the subspaces $G_{k}$ together with the induced fibrations

$$
T \longrightarrow R_{k} \longrightarrow G_{k} .
$$

The fibration in Theorem 3.8 provides the case $k=0$. These fibrations will be induced from corresponding fibrations

$$
\Omega S^{2 n+1}\left\{p^{r}\right\} \longrightarrow E_{k} \longrightarrow G_{k}
$$


as in Theorem 3.8. The entire induction involves obtaining key properties of the skeleta of $\Sigma T$ as well as $G$ and involves a cyclical induction through 14 steps.

Proposition 4.1 As an algebra, $H^{*}(T ; \mathbb{Z} / p \mathbb{Z})$ is generated by classes $u$ of dimension $2 n-1$ and $v_{i}$ of dimension $2 n p^{i}$ for each $i \geq 0$ subject to the relations $v_{i}^{p}=0$ and $u^{2}=0$. For each $i$ define

$$
u_{i}=u v_{0}^{p-1} v_{1}^{p-1} \cdots v_{i-1}^{p-1} .
$$

Then $\beta^{(r+i)} u_{i}=v_{i}$. As a vector space $\widetilde{H}^{*}(T ; \mathbb{Z} / p \mathbb{Z})$ is generated by classes $v(m)$ of dimension $2 m n$ and $u(m)$ of dimension $2 m n-1$ for each $m \geq 1$ where

$$
\begin{aligned}
& v(m)=v_{s}^{e_{s}} \cdots v_{t}^{e_{t}}=\beta^{(r+s)} u(s) \\
& u(m)=u_{s} v_{s}^{e_{s}} v_{s+1}^{e_{s+1}} \cdots v_{t}^{e_{t}}
\end{aligned}
$$

and $m=\sum_{i=s}^{t} e_{i} p^{i}, 0 \leq e_{i}<p, e_{s} \neq 0$.

Proof We apply the Serre spectral sequence for the cohomology of the fibration

$$
S^{2 n-1} \longrightarrow T \longrightarrow \Omega S^{2 n+1} \text {. }
$$

Using $\mathbb{Z} / p \mathbb{Z}$ coefficients we see that

$$
H^{*}(T ; \mathbb{Z} / p \mathbb{Z}) \cong H^{*}\left(S^{2 n-1} ; \mathbb{Z} / p \mathbb{Z}\right) \otimes H^{*}\left(\Omega S^{2 n+1} ; \mathbb{Z} / p \mathbb{Z}\right)
$$

as algebras. Using integer coefficients we see that $v(m)$ is the reduction of a class of order $p^{r+s}$ so $v(m)=\beta^{(r+s)} u(s) \neq 0$. We define $v_{s}=\beta^{(r+s)} u_{s}$.

Note that dually the homology of $T$ has a very simple description. There is a Hopf algebra isomorphism

$$
H_{*}(T) \cong \Lambda(\bar{u}) \otimes \mathbb{Z} / p \mathbb{Z}[\bar{v}]
$$

where $\bar{u}$ and $\bar{v}$ are dual to $u$ and $v$ respectively, and the dual Bocksteins are determined by $\beta^{(r+i)} \bar{v}^{p^{i}}=\bar{u} \bar{v}^{p^{i}-1}$ for $i \geq 0$.

Anick [1] introduced the notation $\mathcal{W}_{a}^{b}$ for the collection of all spaces that are the homotopy type of simply connected locally finite wedges of mod $-p^{s}$ Moore spaces for $a \leq s \leq b$. Note that any simply connected Moore space is a suspension, so any space in $\mathcal{W}_{a}^{b}$ is a suspension. Recall that the smash of two Moore spaces is homotopy equivalent to a wedge of Moore spaces: if $s \leq t$ then there is a homotopy equivalence

$$
P^{m}\left(p^{s}\right) \wedge P^{n}\left(p^{t}\right) \simeq P^{m+n}\left(p^{s}\right) \wedge P^{m+n-1}\left(p^{s}\right) .
$$

In particular, $\mathcal{W}_{a}^{b}$ is closed under smash products. Recall also that any retract of a wedge of Moore spaces is homotopy equivalent to a wedge of Moore spaces, so $\mathcal{W}_{a}^{b}$ is closed under retracts. 
Lemma 4.2 Suppose $W \in \mathcal{W}_{a}^{b}$ is simply connected and $f: P^{k}\left(p^{t}\right) \longrightarrow W$ is divisible by $p^{b}$.

(a) Write $W=W_{1} \vee W_{2}$ with $W_{1} \in \mathcal{W}_{a}^{b-1}$ and $W_{2} \in \mathcal{W}_{b}^{b}$. Then $f$ factors through $W_{2}$ up to homotopy.

(b) Suppose in addition that $W_{2}$ is $(d-1)$ connected and $k<p d$. Then $f \sim *$.

Proof Since $W$ is a wedge, there is a homotopy equivalence

$$
\Omega W=\Omega W_{2} \times \Omega\left(W_{1} \rtimes \Omega W_{2}\right)
$$

(see, for example, the first author's work [7]). Since $W_{1}, W_{2} \in \mathcal{W}_{a}^{b}$, both spaces are suspensions, and we can write $W_{1}=\Sigma \bar{W}_{1}$ and $W_{2}=\Sigma \bar{W}_{2}$. Since $W_{1}$ is a suspension, we have $W_{1} \rtimes \Omega W_{2} \simeq W_{1} \vee\left(W_{1} \wedge \Omega W_{2}\right)$. For the right wedge summand, the James splitting of $\Sigma \Omega \Sigma X$ as $\bigvee \Sigma X^{(i)}$ gives

$$
W_{1} \wedge \Omega W_{2} \simeq \Sigma \bar{W}_{1} \wedge \Omega \Sigma \bar{W}_{2} \simeq \bar{W}_{1} \wedge\left(\bigvee \Sigma \bar{W}_{2}^{(i)}\right)
$$

Combining, we have

$$
W_{1} \rtimes \Omega W_{2} \simeq W_{1} \vee\left(W_{1} \wedge\left(\bigvee W_{2}^{(i)}\right)\right) .
$$

In particular, since $\mathcal{W}_{a}^{b}$ is closed under smash products, we have $W_{1} \rtimes \Omega W_{2} \in \mathcal{W}_{a}^{b}$. Applying the Hilton-Milnor theorem therefore implies that

$$
\Omega\left(W_{1} \rtimes \Omega W_{2}\right) \simeq \prod_{i} \Omega P^{n_{i}}\left(p^{s_{i}}\right)
$$

with $a \leq s \leq b-1$.

By [16], the $p^{r+1}$-power map on $\Omega^{2} P^{m}\left(p^{r}\right)$ is null homotopic for any $r \geq 1$ and $m \geq 3$. Thus $P^{m}\left(p^{r}\right)$ admits no nontrivial maps which are divisible by $p^{r+1}$. In our case, this implies that $\prod_{i} \Omega P^{n_{i}}\left(p^{s_{i}}\right)$ admits no nontrivial maps which are divisible by $p^{b}$. Thus the adjoint of $f$, which is divisible by $p^{b}$, is trivial on $\Omega\left(W_{1} \rtimes \Omega W_{2}\right)$ and so factors through the inclusion $\Omega W_{2} \longrightarrow \Omega W$. Hence, taking the adjoint, $f$ factors through the inclusion $W_{2} \longrightarrow W$, proving part (a).

For part (b), since $W_{2} \in \mathcal{W}_{b}^{b}$ and $W_{2}$ is $(d-1)$-connected, the Hilton-Milnor theorem implies that $\Omega W_{2}=\prod \Omega P^{n_{i}}\left(p^{b}\right)$ where $n_{i}>d$. By [6;16], $P^{2 m+1}\left(p^{r}\right)$ admits no nontrivial maps which are divisible by $p^{r}$ from a CW-complex of dimension $t<2 m p$, and $\left.P^{2 m}\left(p^{b}\right)\right)$ admits no nontrivial maps which are divisible by $p^{r}$ from a $\mathrm{CW}$-complex of dimension $t<2(2 m-1) p$. In our case, the CW-complex is $P^{k}\left(p^{t}\right)$, the domain of $f$, and the target Moore spaces are the $P^{n_{i}}\left(p^{b}\right)$ in the decomposition of $\Omega W_{2}$. Since $n_{i}>d$ for each $i$, the hypothesis $k<p d$ guarantees that the component of $f$ on $P^{n_{i}}\left(p^{b}\right)$, being divisible by $p^{b}$, is null homotopic. So $f$ is null homotopic. 
Theorem 4.3 For each $k \geq 0$ there are spaces $G_{k}$ and $W_{k} \in \mathcal{W}_{r}^{r+k-1}$ satisfying the following conditions:

(a) $\Sigma T^{2 n p^{k}-2} \simeq G_{k-1} \vee W_{k}$.

(b) There are maps $g_{k}: T^{2 n p^{k}-2} \longrightarrow \Omega G_{k-1}$ and $h_{k-1}: \Omega G_{k-1} \longrightarrow T$ such that $h_{k-1} g_{k}$ is homotopic to the inclusion of $T^{2 n p^{k}-2}$ into $T$.

(c) There is a homotopy commutative diagram of cofibration sequences which defines the space $G_{k}$

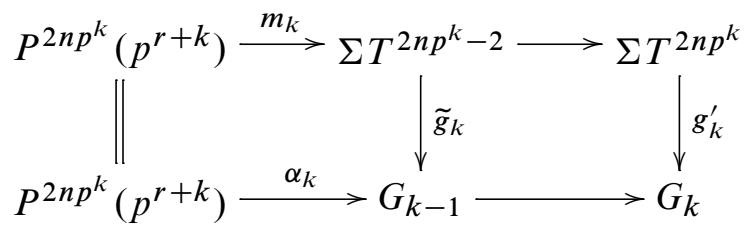

where $\tilde{g}_{k}$ is the adjoint of $g_{k}$.

(d) There is a map e: $P^{2 n p^{k}}\left(p^{r+k-1}\right) \vee P^{2 n p^{k}+1}\left(p^{r+k-1}\right) \longrightarrow \Sigma T^{2 n p^{k}}$ which induces an epimorphism in mod-p homology in dimensions $2 n p^{k}$ and $2 n p^{k}+1$.

(e) The map $m_{k}: P^{2 n p^{k}}\left(p^{r+k}\right) \longrightarrow \Sigma T^{2 n p^{k}-2}$ is divisible by $p^{r+k-1}$.

(f) There is a map $\varphi_{k}: G_{k} \longrightarrow S^{2 n+1}\left\{p^{r}\right\}$ extending $\varphi_{k-1}$.

(g) $\Sigma G_{k} \in \mathcal{W}_{r}^{r+k}$.

(h) There is a homotopy commutative diagram of fibration sequences

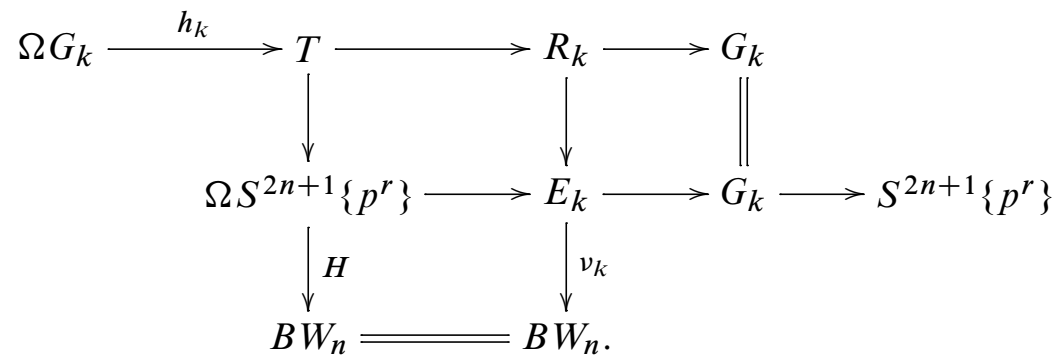

(i) $\Sigma^{2} \Omega G_{k-1} \in \mathcal{W}_{r}^{r+k-1}$.

(j) The equivalence in (a) extends to an equivalence $\Sigma T^{2 n p^{k}} \simeq G_{k} \vee W_{k}$.

(k) $\Sigma^{2} T^{2 n p^{k}} \in \mathcal{W}_{r}^{r+k}$.

(1) $G_{k} \wedge T^{2 n p^{k}} \in \mathcal{W}_{r}^{r+k}$.

(m) $\Sigma T^{2 n p^{k}} \wedge T^{2 n p^{k}} \in \mathcal{W}_{r}^{r+k}$. 
(n) There is a map $\mu_{k}: T^{2 n p^{k}} \times T \longrightarrow T$ which is the inclusion on the first axis and the identity on the second. Furthermore there is a homotopy commutative square

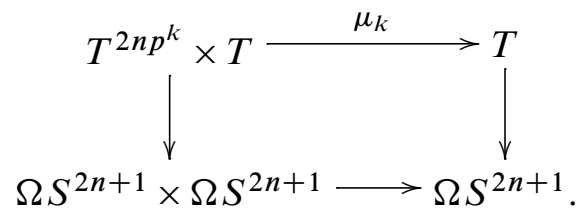

Proof With $G_{-1}=*$ and $G_{0}=P^{2 n+1}$ these statements are all immediate for $k=0$ with $\varphi_{0}: P^{2 n+1} \longrightarrow S^{2 n+1}\left\{p^{r}\right\}$ the inclusion, $E_{0}=E$ from Theorem 3.6, $v_{0}=v^{E}$, $\mu_{0}: P^{2 n} \times T \longrightarrow T$ obtained from the action of $\Omega P^{2 n+1}$ on $T$ defined by the fibration in Theorem 3.6. We now supposed that (a)-(n) are all valid with $k-1$ in the place of $k$ and we proceed to prove them for $k$.

Proof of (a) We will construct a map

$$
f_{m}: P^{2 m n+1}\left(p^{r+s}\right) \longrightarrow \Sigma T^{2 n p^{k}-2}
$$

which induces a monomorphism in mod- $p$ homology for each $m$ satisfying $p^{k-1}<$ $m<p^{k}$, where $s=v_{p}(m)$. We then assemble these into a map

$$
\Sigma T^{2 n p^{k-1}} \vee\left(\bigvee_{m=p^{k-1}+1}^{p^{k}-1} P^{2 m n+1}\left(p^{r+s}\right)\right) \longrightarrow \Sigma T^{2 n p^{k}-2}
$$

which induces an isomorphism in mod- $p$ homology. By applying (j) in the case $k-1$ we are done.

To construct the maps $f_{m}$ we appeal to (n) in the case $k-1$ and iterate this to produce a diagram with $p$ factors

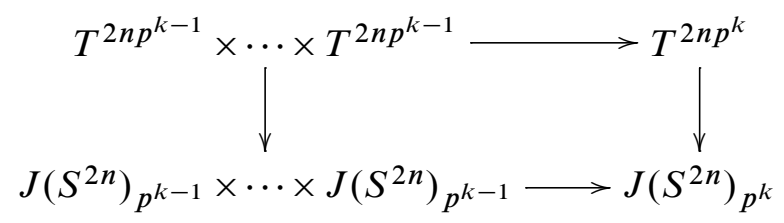

where $J\left(S^{2 n}\right)_{j}$ is the $2 n j$ skeleton of $\Omega S^{2 n+1}$. Write $m$ to the base $p$. Since $p^{k-1}<m<p^{k}, m$ has coefficients of $p^{i}$ for $i<k$ and the coefficient of $p^{k-1}$ is not zero

$$
m=a_{s} p^{s}+\cdots+a_{k-1} p^{k-1} \text { with } a_{s}>0 \text { and } a_{k-1}>0
$$


Let $l=a_{s} p^{s}+\cdots+a_{k-2} p^{k-2}$ so that $m=l+a_{k-1} p^{k-1}$ and further restrict the above diagram to one with $a_{k-1}+1$ factors

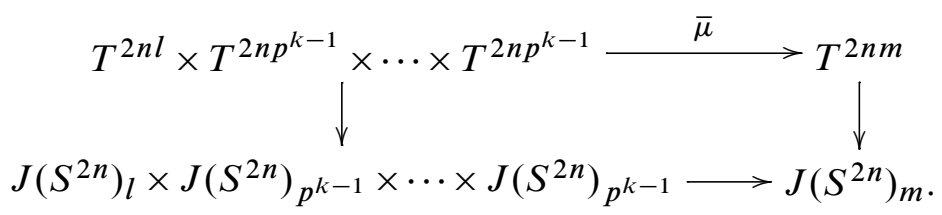

Applying the maps in this diagram to a generator of $H^{2 m n}\left(J\left(S^{2 n}\right)_{m} ; \mathbb{Z} / p \mathbb{Z}\right)$ we get

$$
(\bar{\mu})^{*}(v(m))=v(l) \otimes v_{k-1} \otimes \cdots \otimes v_{k-1} .
$$

Now $v(m)=\beta^{(r+s)} u(m)$ and $v(l)=\beta^{(r+s)} u(l)$, so

$$
v(l) \otimes v_{k-1} \otimes \cdots \otimes v_{k-1}=\beta^{(r+s)}\left(u(l) \otimes v_{k-1} \otimes \cdots \otimes v_{k-1}\right) .
$$

Applying (a) and (m) in case $k-1$ we see that

$$
\Sigma\left(T^{2 n l} \times T^{2 n p^{k-1}} \times \cdots \times T^{2 n p^{k-1}}\right) \simeq V \vee W
$$

where $V$ is a wedge of $p$ spaces, all but one being $G_{k-1}$ with the other being the subspace $G_{l}$ of $G_{k-1}$, and $W \in \mathcal{W}_{r}^{r+k-1}$. In particular, the dimension of $V$ is less than $2 n p^{k-1}+2$. Since $p^{k-1}<m, \mu^{*}(u(m))$ projects trivially to $H^{*}(v)$. But since $W \in \mathcal{W}_{r}^{r+k-1}$, for any class $\xi \in H^{i}(W ; \mathbb{Z} / p \mathbb{Z})$ with $\beta^{(j)} \xi \neq 0$, there is a map

$$
f_{\xi}: P^{i+1}\left(p^{j}\right) \longrightarrow W
$$

with $f_{\xi}^{*}$ an epimorphism. Thus for each $m$ satisfying $p^{k-1}<m<p^{k}$ we may choose such a map corresponding to $\xi=u(l) \otimes v_{k-1} \otimes \cdots \otimes v_{k-1}$. The composition

$$
P^{2 m n+1}\left(p^{r+s}\right) \stackrel{f_{\xi}}{\longrightarrow} \Sigma\left(T^{2 n l} \times T^{2 n p^{k-1}} \times \cdots \times T^{2 n p^{k-1}}\right) \stackrel{\Sigma \bar{\mu}}{\longrightarrow} \Sigma T^{2 m n}
$$

therefore gives the desired map $f_{m}$.

Proof of (b) From part (a) we obtain a map $T^{2 n p^{k}-2} \longrightarrow \Omega G_{k-1}$ which induces an isomorphism in $\pi_{2 n-1}()$. The composition

$$
T^{2 n p^{k}-2} \longrightarrow \Omega G_{k-1} \stackrel{h_{k-1}}{\longrightarrow} T
$$

factors through the inclusion of $T^{2 n p^{k}-2}$ and provides a self map of $T^{2 n p^{k}-2}$ which induces an isomorphism on $\pi_{2 n-1}()$. Calculations with cup products and Bocksteins show that this map is a homotopy equivalence, so composing with the inverse provides a possibly different map

$$
g_{k}: T^{2 n p^{k}-2} \longrightarrow \Omega G_{k-1}
$$

such that $h_{k-1} g_{k}$ is homotopic to the inclusion. 
Proof of (c) Using the map $g_{k}$ from (b) we construct a commutative diagram where the bottom row is the fibration sequence from (h) in case $k-1$ and the middle row is a cofibration sequence

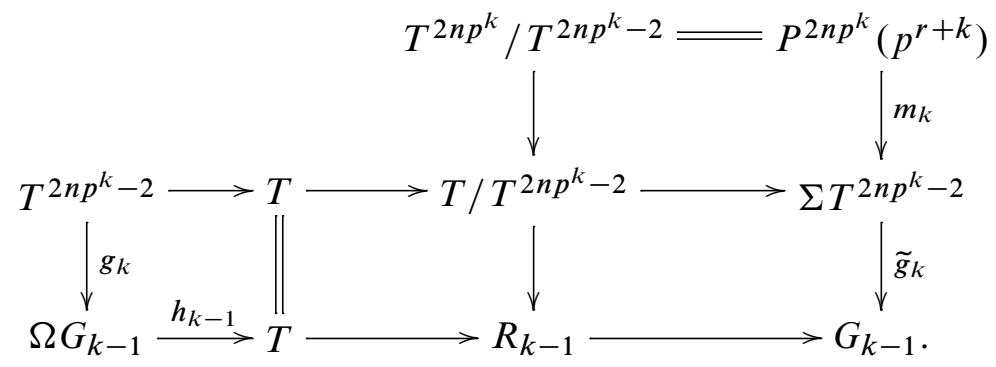

Here, $m_{k}$ is defined as the composite $T^{2 n p^{k}} / T^{2 n p^{k}-2} \rightarrow T / T^{2 n p^{k}-2} \rightarrow \Sigma T^{2 n p^{k}-2}$. Define

$$
\alpha_{k}: P^{2 n p^{k}}\left(p^{r+k}\right) \longrightarrow G_{k-1}
$$

as the composite $\tilde{g}_{k} \circ m_{k}$. Define $g_{k}^{\prime}$ by the diagram of cofibration sequences

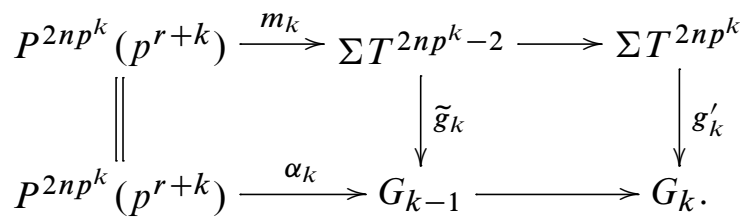

Proof of (d) As in part (a), we consider the diagram

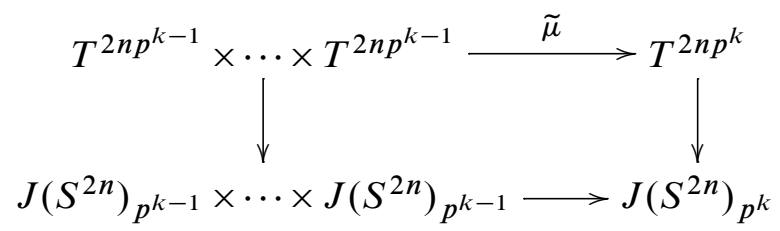

with $p$ factors on the left. This is defined by iterated application of part (n) in case $k-1$. Clearly

$$
\tilde{\mu}^{*}\left(v_{k}\right)=v_{k-1} \otimes \cdots \otimes v_{k-1}=\beta^{(r+k-1)}\left(u v_{1}^{p-1} \cdots v_{k-2}^{p-1} \otimes v_{k-1} \otimes \cdots \otimes v_{k-1}\right) .
$$

As before there is a map

$$
q: P^{2 n p^{k}+1}\left(p^{r+k-1}\right) \longrightarrow \Sigma\left(T^{2 n p^{k-1}} \times \cdots \times T^{2 n p^{k-1}}\right)
$$


such that $(\Sigma(\tilde{\mu}) q)_{*}$ is an epimorphism in $\mathbb{Z} / p \mathbb{Z}$ homology in dimension $2 n p^{k}+1$, obtained by applying (k) and (m) in case $k-1$. Similarly, $v_{k}=\beta^{(r+k)} u_{k}$ and

$$
(\tilde{\mu})^{*} u_{k}=\sum_{p \text { terms }} v_{k-1} \otimes \cdots \otimes v_{k-1} \otimes u_{k-1} \otimes v_{k-1} \cdots \otimes v_{k-1} .
$$

In particular, the map

$$
T^{2 n p^{k-1}-1} \times T^{2 n p^{k-1}} \times \cdots \times T^{2 n p^{k-1}} \stackrel{\tilde{\mu}^{\prime}}{\longrightarrow} T^{2 n p^{k}}
$$

has the property that

$$
\begin{aligned}
\left(\tilde{\mu}^{\prime}\right)^{*}\left(u_{k}\right) & =u_{k-1} \otimes v_{k-1} \otimes \cdots \otimes v_{k-1} \\
& =\beta^{(r+k-1)}\left(u_{k-1} \otimes u_{k-1} \otimes v_{k} \otimes \cdots \otimes v_{k}\right) .
\end{aligned}
$$

It follows, as before, that there is a map

$$
r: P^{2 n p^{k}}\left(p^{r+k-1}\right) \longrightarrow \Sigma\left(T^{2 n p^{k-1}-1} \times T^{2 n p^{k-1}} \times \cdots \times T^{2 n p^{k-1}}\right)
$$

such that $\left(\Sigma\left(\tilde{\mu}^{\prime}\right) r\right)_{*}$ is an epimorphism in $\mathbb{Z} / p \mathbb{Z}$ homology in dimension $2 n p^{k}$. We construct $e$ as the wedge sum

$$
e=\left(\Sigma \tilde{\mu}^{\prime}\right) r \vee(\Sigma \tilde{\mu}) q: P^{2 n p^{k}}\left(p^{r+k-1}\right) \vee P^{2 n p^{k}+1}\left(p^{r+k-1}\right) \longrightarrow \Sigma T^{2 n p^{k}} .
$$

Proof of (e) We will construct a map $n_{k}: P^{2 n p^{k}}\left(p^{r+k}\right) \rightarrow \Sigma T^{2 n p^{k}-2}$ such that $m_{k} \sim p^{r+k-1} n_{k}$ out of a commutative diagram obtained from mapping a cofibration sequence into a fibration sequence. Given a Hurewicz fibration

$$
F \stackrel{\iota}{\rightarrow} E \stackrel{\pi}{\rightarrow} B
$$

and a commutative square

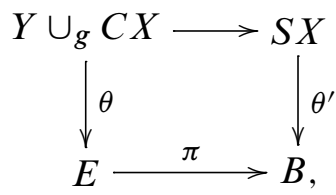

one can construct a commutative ladder

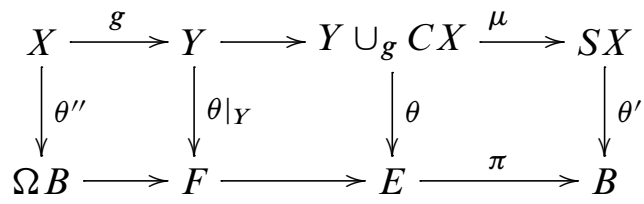


where $\theta^{\prime \prime}$ is adjoint to $\theta^{\prime}$. (See, for example, Neisendorfer [17, 3.3.1]). We will apply this to the cofibration induced by the map

$$
P^{2 n p^{k}+1}\left(p^{r+k}\right) \stackrel{p^{r+k-1}}{\longrightarrow} P^{2 n p^{k}+1}\left(p^{r+k}\right)
$$

and the fibration induced by the map

$$
\Sigma T^{2 n p^{k}} \stackrel{\pi}{\rightarrow} P^{2 n p^{k}+1}\left(p^{r+k}\right)
$$

pinching the $2 n p^{k}-1$ skeleton to a point. In this case the space $Y \cup_{g} C X$ is homotopy equivalent to $P^{2 n p^{k}}\left(p^{r+k-1}\right) \vee P^{2 n p^{k}+1}\left(p^{r+k-1}\right)$ and the map $\mu_{*}$ induces an epimorphism in $\bmod p$ homology. By part $(\mathrm{d}),(\pi e)_{*}$ induces a $\bmod p$ homology epimorphism, so for some equivalence $\xi, \pi e \xi \sim \mu$. Taking $\theta=e \xi$ and $\theta^{\prime}=1$, we obtain the diagram

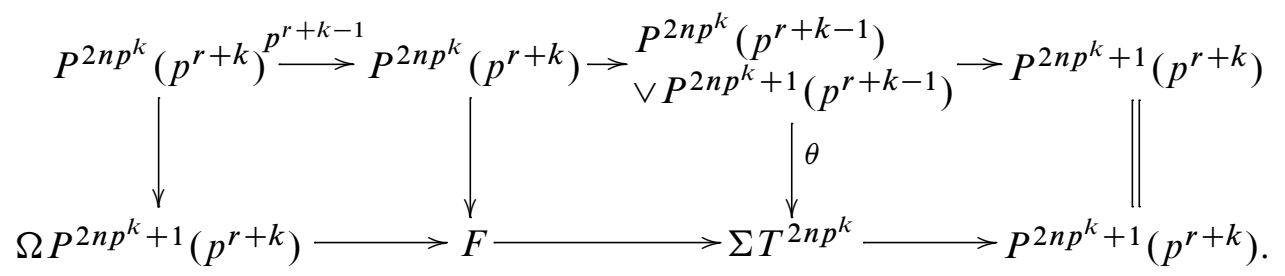

The $2 n p^{k}+2 n-1$ skeleton of $F$ is $\Sigma T^{2 n p^{k}-2}$. Restricting the lefthand square to the $2 n p^{k}+1$ skeleton yields a homotopy commutative square:

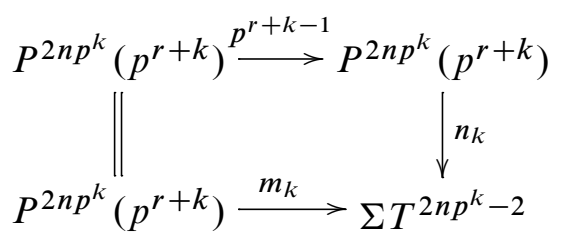

Proof of (f) To show that there is an extension of $\varphi_{k-1}$ to $\varphi_{k}$,

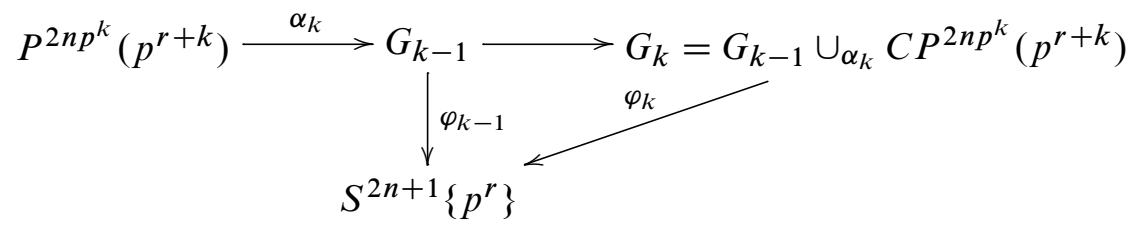

it suffices to show that $\alpha_{k}$ is divisible by $p^{r}$. This holds by (e) since $r+k-1 \geq r$.

Proof of (g) By part (g) for $k-1$, there is a homotopy equivalence $\Sigma G_{k-1} \simeq$ $\bigvee_{i=0}^{k-1} P^{2 n p^{i}+2}\left(p^{r+i}\right)$. Also, by definition, $\Sigma G_{k}=\Sigma G_{k-1} \cup \Sigma \alpha_{k} C P^{2 n p^{k}}\left(p^{r+k}\right)$. 
By part (e) $\alpha_{k}=\tilde{\alpha} \circ\left(p^{r+k-1} \iota\right)$ so $\Sigma \alpha_{k}=\Sigma \widetilde{\alpha} \circ\left(p^{r+k-1} \iota\right) \sim\left(p^{r+k-1} \iota\right) \circ \Sigma \widetilde{\alpha}$. However

$$
p^{r+k-1} \iota: \bigvee_{i=0}^{k-1} P^{2 n p^{i}+2}\left(p^{r+i}\right) \rightarrow \bigvee_{i=0}^{k-1} P^{2 n p^{i}+2}\left(p^{r+i}\right)
$$

is null homotopic since the order of the identity map on a mod $-p^{r}$ Moore space is $p^{r}$.

Proof of (h) In case $k=0$, this is Theorem 3.8. As we have constructed $\varphi_{k}: G_{k} \longrightarrow$ $S^{2 n+1}\left\{p^{r}\right\}$ in part (f), we have a pullback diagram of principal fibrations

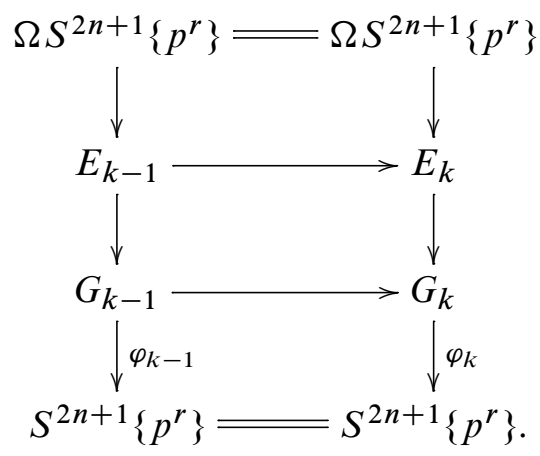

Let $\left(C P^{2 n p^{k}}\left(p^{r+k}\right), P^{2 n p^{k}}\left(p^{r+k}\right)\right) \stackrel{\theta}{\longrightarrow}\left(G_{k}, G_{k-1}\right)$ be the relative homeomorphism extending $\alpha_{k}$ and defining $G_{k}$. Since $E_{k} \longrightarrow G_{k}$ has the homotopy lifting property, we can find a map $\phi$ : $C P^{2 n p^{k}}\left(p^{r+k}\right) \longrightarrow E_{k}$ covering $\theta$. As $E_{k-1}$ is a pullback, we get a map of pairs

$$
\phi:\left(C P^{2 n p^{k}}\left(p^{r+k}\right), P^{2 n p^{k}}\left(p^{r+k}\right)\right) \longrightarrow\left(E_{k}, E_{k-1}\right)
$$

covering $\theta$. The composition

$$
P^{2 n p^{k}}\left(p^{r+k}\right) \stackrel{\phi}{\longrightarrow} E_{k-1} \longrightarrow G_{k-1}
$$

is $\alpha_{k}$ which is divisible by $p^{r+k-1}$. Since $k \geqslant 1$ we may apply Extension Theorem 2.2 to obtain an extension $v_{k}$ of $v_{k-1}$.

Proof of (i) By part (j) in case $k-1, \Sigma^{2} \Omega G_{k-1}$ is a retract of $\Sigma^{2} \Omega \Sigma T^{2 n p^{k-1}}$. The latter space splits since the loop space can be approximated by the James construction [12], giving

$$
\Sigma^{2} \Omega \Sigma T^{2 n p^{k-1}} \simeq \Sigma^{2}\left(\bigvee_{i \geq 1}\left(T^{2 n p^{k-1}}\right)^{(i)}\right)
$$

which is in $\mathcal{W}_{r}^{r+k-1}$ by (k) and (l) in case $k-1$. Since $\mathcal{W}_{r}^{r+k-1}$ is closed under retracts we are done. 
Proof of (j) By part (a), we have $\Sigma T^{2 n p^{k}-2} \simeq G_{k-1} \vee W_{k}$ and by (e), we have

$$
\Sigma T^{2 n p^{k}} \simeq\left(\Sigma T^{2 n p^{k}-2}\right) \cup_{m_{k}} C P^{2 n p^{k}}\left(p^{r+k}\right)
$$

with $m_{k}$ divisible by $p^{r+k-1}$. It suffices to show that the map

$$
m_{k}: P^{2 n p^{k}}\left(p^{r+k}\right) \longrightarrow \Sigma T^{2 n p^{k}-2} \simeq G_{k-1} \vee W_{k}
$$

factors though $G_{k-1}$. To this end, observe that there is a homotopy decomposition

$$
\Omega\left(G_{k-1} \vee W_{k}\right) \simeq \Omega G_{k-1} \times \Omega\left(W_{k} \rtimes \Omega G_{k-1}\right) .
$$

We will show that any map $P^{2 n p^{k}}\left(p^{r+k}\right) \longrightarrow W_{k} \rtimes \Omega G_{k-1}$ which is divisible by $p^{r+k-1}$ is null homotopic. Since $W_{k}$ is $(4 n-1)$-connected, the Moore spaces in $W_{k}$ are double suspensions, so $W_{k} \rtimes \Omega G_{k-1} \in \mathcal{W}_{r}^{r+k-1}$ by (i). In fact, $W_{k} \rtimes \Omega G_{k-1} \simeq$ $W_{1} \vee W_{2}$ with $W_{1} \in \mathcal{W}_{r}^{r+k-2}$ and $W_{2}$ a retract of

$$
\bigvee_{r=2}^{p-1} P^{2 n p^{k-1}+1}\left(p^{r+k-1}\right) \rtimes \Omega G_{k-1}
$$

which is $4 n p^{k-1}-1$ connected. The result follows from Lemma 4.2.

Proof of (k) This follows immediately from (g) and (j).

Proof of (1) This follows from 3 steps based on an analysis which first appeared in [18].

Step $1 G_{k} \wedge T^{2 n p^{k-1}} \in \mathcal{W}_{r}^{r+k-1}$.

Consider the cofibration sequence

$$
P^{2 n p^{k}}\left(p^{r+k}\right) \wedge T^{2 n p^{k-1}} \stackrel{\alpha_{k} \wedge 1}{\longrightarrow} G_{k-1} \wedge T^{2 n p^{k-1}} \rightarrow G_{k} \wedge T^{2 n p^{k-1}} .
$$

We have $P^{2 n p^{k}}\left(p^{r+k}\right) \wedge T^{2 n p^{k-1}} \in \mathcal{W}_{r}^{r+k-1}$ and $\alpha_{k} \wedge 1$ is divisible by $p^{r+k-1}$. Consequently, $\alpha_{k} \wedge 1 \sim *$ and so there is a homotopy decomposition

$$
G_{k} \wedge T^{2 n p^{k-1}} \simeq\left(G_{k-1} \wedge T^{2 n p^{k-1}}\right) \vee\left(P^{2 n p^{k}+1}\left(p^{r+k}\right) \wedge T^{2 n p^{k-1}}\right)
$$

which is in $\mathcal{W}_{r}^{r+k-1}$ by (k) in case $k-1$.

Step $2 G_{k-1} \wedge T^{2 n p^{k}} \in \mathcal{W}_{r}^{r+k-1}$.

By (j) in case $k-1, G_{k-1} \wedge T^{2 n p^{k}}$ is a retract of $\Sigma T^{2 n p^{k-1}} \wedge T^{2 n p^{k}}$. But

$$
\Sigma T^{2 n p^{k-1}} \wedge T^{2 n p^{k}} \simeq T^{2 n p^{k-1}} \wedge \Sigma T^{2 n p^{k}} \simeq T^{2 n p^{k-1}} \wedge\left(G_{k} \vee W_{k}\right)
$$


by (j). By Step 1 and (k) in case $k-1$, the latter space is in $\mathcal{W}_{r}^{r+k-1}$. Since $\mathcal{W}_{r}^{r+k-1}$ is closed under retracts, we therefore have $G_{k-1} \wedge T^{2 n p^{k}} \in \mathcal{W}_{r}^{r+k-1}$.

Step $3 G_{k} \wedge T^{2 n p^{k}} \in \mathcal{W}_{r}^{r+k}$.

Consider here the cofibration sequence

$$
P^{2 n p^{k}}\left(p^{r+k}\right) \wedge T^{2 n p^{k}} \stackrel{\alpha_{k} \wedge 1}{\longrightarrow} G_{k-1} \wedge T^{2 n p^{k}} \rightarrow G_{k} \wedge T^{2 n p^{k}} .
$$

The first space is in $\mathcal{W}_{r}^{r+k}$ by $(\mathrm{k})$ and the second is in $\mathcal{W}_{r}^{r+k-1}$ by Step 2 . In fact, $G_{k-1} \wedge T^{2 n p^{k}} \simeq\left(P^{2 n p^{k-1}+1}\left(p^{r+k-1}\right) \wedge T^{2 n p^{k}}\right) \vee W^{\prime}$ with $W^{\prime} \in \mathcal{W}_{r}^{r+k-2}$. Here, the projection onto the first factor is $\rho_{k-1} \wedge 1$, where $\rho_{k-1}$ is obtained by collapsing $G_{k-2}$ to a point. Applying Lemma 4.2(b), we see that if $\alpha_{k} \wedge 1$ is nontrivial, so is the composition

$$
P^{2 n p^{k}}\left(p^{r+k}\right) \wedge T^{2 n p^{k}} \stackrel{\alpha_{k} \wedge 1}{\longrightarrow} G_{k-1} \wedge T^{2 n p^{k}} \stackrel{\rho_{k-1} \wedge 1}{\longrightarrow} P^{2 n p^{k-1}}\left(p^{r+k-1}\right) \wedge T^{2 n p^{k}} .
$$

We will show that this composition is null homotopic. Let $\delta=\rho_{k-1} \alpha_{k}$, which is divisible by $p^{r+k-1}$ because $\alpha_{k}$ is. According to [15], the $p^{r+k-1}$-power map on $S^{2 n p^{k-1}+1}\left\{p^{r+k-1}\right\}$ is null homotopic. Therefore the composition

$$
P^{2 n p^{k}}\left(p^{r+k}\right) \stackrel{\delta}{\longrightarrow} P^{2 n p^{k-1}+1}\left(p^{r+k-1}\right) \longrightarrow S^{2 n p^{k-1}+1}\left\{p^{r+k-1}\right\}
$$

is null homotopic. It follows that the composition

$$
P^{2 n p^{k}}\left(p^{r+k}\right) \stackrel{\delta}{\longrightarrow} P^{2 n p^{k-1}+1}\left(p^{r+k-1}\right) \stackrel{\rho}{\longrightarrow} S^{2 n p^{k-1}+1}
$$

is null homotopic. Since the map

$$
P^{2 n p^{k-1}+1}\left(p^{r+k}\right) \wedge T^{2 n p^{k}} \stackrel{\rho \wedge 1}{\longrightarrow} S^{2 n p^{k-1}+1} \wedge T^{2 n p^{k}}
$$

has a left homotopy inverse, the map

$$
P^{2 n p^{k}}\left(p^{r+k}\right) \wedge T^{2 n p^{k}} \stackrel{\delta \wedge 1}{\longrightarrow} P^{2 n p^{k-1}+1}\left(p^{r+k}\right) \wedge T^{2 n p^{k}}
$$

is null homotopic. Since $\alpha_{k} \wedge 1$ is the composition

$$
P^{2 n p^{k}}\left(p^{r+k}\right) \wedge T^{2 n p^{k}} \stackrel{\alpha \wedge 1}{\longrightarrow} G_{k-1} \wedge T^{2 n p^{k}} \stackrel{\delta \wedge 1}{\longrightarrow} P^{2 n p^{k-1}+1}\left(p^{r+k}\right) \wedge T^{2 n p^{k}},
$$

it is null homotopic as well. Consequently, there is a homotopy decomposition

$$
G_{k} \wedge T^{2 n p^{k}} \simeq\left(G_{k-1} \wedge T^{2 n p^{k}}\right) \vee\left(P^{2 n p^{k}+1}\left(p^{r+k}\right) \wedge T^{2 n p^{k}}\right) .
$$

Both terms on the right are in $\mathcal{W}_{r}^{r+k}$ by (k) and Step 2 . 
Proof of (m) By (j), $\Sigma T^{2 n p^{k}} \wedge T^{2 n p^{k}} \simeq\left(G_{k} \vee W_{k}\right) \wedge T^{2 n p^{k}}$. By (l), $G_{k} \wedge T^{2 n p^{k}} \in$ $\mathcal{W}_{r}^{r+k}$, and as $W_{k}$ is a wedge of Moore spaces which are at least $(4 n-1)$-connected, it is a double suspension, so $W_{k} \wedge T^{2 n p^{k}} \in \mathcal{W}_{r}^{r+k}$ by (k). So $\Sigma T^{2 n p^{k}} \wedge T^{2 n p^{k}} \in$ $\mathcal{W}_{r}^{r+k}$.

Proof of (n) Since the composite $R_{k} \longrightarrow E_{k} \longrightarrow G_{k} \longrightarrow S^{2 n+1}\left\{p^{r}\right\} \longrightarrow S^{2 n+1}$ is null homotopic by $(h)$, there is a commutative diagram of principal fibrations

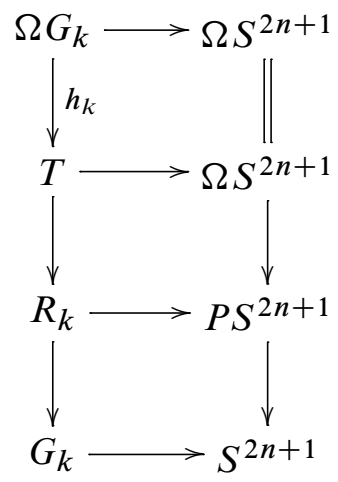

where $P S^{2 n+1}$ is the path space on $S^{2 n+1}$. Consequently the actions are compatible:

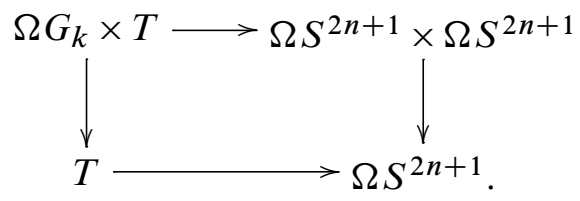

Using (j) we construct a map $g_{k}: T^{2 n p^{k}} \longrightarrow \Omega G_{k}$ such that the composition

$$
T^{2 n p^{k}} \stackrel{g_{k}}{\longrightarrow} \Omega G_{k} \stackrel{h_{k}}{\longrightarrow} T
$$

is homotopic to the inclusion as in (b). This gives a homotopy commutative diagram

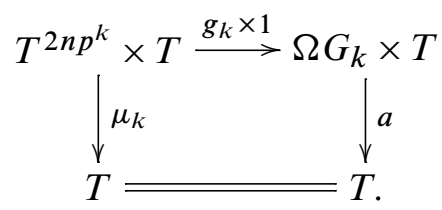

Combining the preceding two diagrams gives the result and completes the induction. $\square$

We now consider the limiting case. Write $G=\bigcup G_{k}, R=\bigcup R_{k}$ and $E_{\infty}=\bigcup E_{k}$. 
Theorem 4.4 There is a diagram of fibration sequences

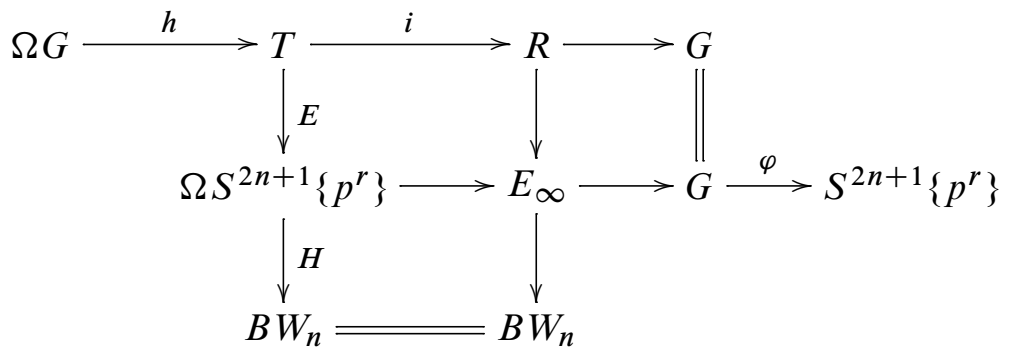

and there are maps $\tilde{g}: T \longrightarrow \Omega G$ and $f: G \longrightarrow \Sigma T$ such that the composites

$$
\begin{aligned}
& G \stackrel{f}{\longrightarrow} \Sigma T \stackrel{g}{\longrightarrow} G \\
& T \stackrel{\text { g }}{\longrightarrow} \Omega G \stackrel{h}{\longrightarrow} T
\end{aligned}
$$

are homotopic to the identity maps.

Proof The diagram is the direct limit of the diagrams in Theorem 4.3 (h) with

$$
h=\underline{\lim } h_{k}, \quad g=\lim _{\longrightarrow} g_{k} \quad \text { and } \quad f=\lim _{\longrightarrow} f_{k},
$$

where $f_{k}: G_{k} \longrightarrow \Sigma T^{2 n p^{k}}$ is a right inverse for $g_{k}$ given by Theorem $4.3(\mathrm{j})$.

Theorem 4.5 The following space belong to $\mathcal{W}_{r}^{\infty}: \Sigma^{2} \Omega G, \Sigma G, G \wedge T, \Sigma T \wedge T$, and $W$ where $\Sigma T \simeq G \vee W$.

Proof This follows immediately from the results in Theorem 4.3 by taking limits. $\square$

The retraction of $T$ off $\Omega G$ in Theorem 4.4 induces an $H$-structure on $T$ by the composite

$$
m: T \times T \stackrel{\widetilde{g} \times \widetilde{g}}{\longrightarrow} \Omega G \times \Omega G \longrightarrow \Omega G \stackrel{h}{\longrightarrow} T .
$$

The following proposition establishes the $H$-fibration property in Theorem 1.1 (a) as a consequence of a slightly stronger result.

Proposition 4.6 The map $T \stackrel{E}{\longrightarrow} \Omega S^{2 n+1}\left\{p^{r}\right\}$ is an $H$ map with respect to the $H-$ space structure $m$ on $T$. Consequently, there is an $H$-fibration sequence $S^{2 n-1} \longrightarrow$ $T \longrightarrow \Omega S^{2 n+1}$. 
Proof Filling in the fibration diagram in Theorem 4.4 on the right, we obtain a homotopy commutative square

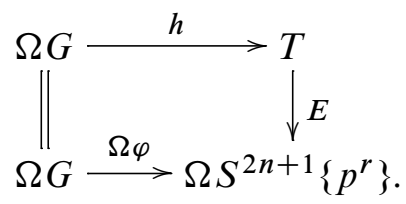

Now consider the diagram

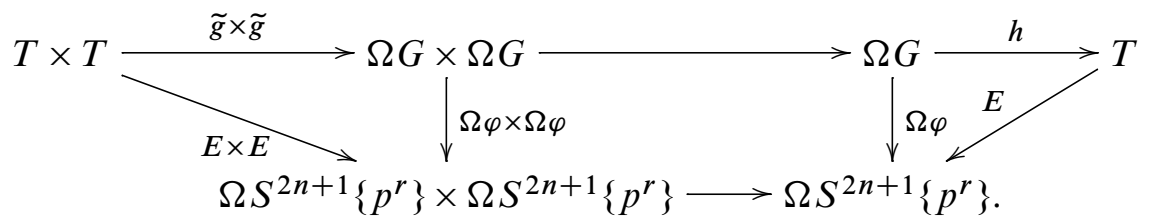

The middle square commutes as $\Omega \varphi$ is an $H$-map and we have just seen that the right triangle commutes. The left triangle commutes since $\varphi \sim E h$, so $\varphi \widetilde{g} \sim E$. As the top row is the definition of the multiplication $m$ on $T$, the commutativity of the diagram implies that $E$ is an $H$-map.

Consequently, the composition $T \stackrel{E}{\longrightarrow} \Omega S^{2 n+1}\left\{p^{r}\right\} \longrightarrow \Omega S^{2 n+1}$ is an $H$-map as it is a composite of $H$-maps, and so the homotopy fibration $S^{2 n-1} \longrightarrow T \longrightarrow \Omega S^{2 n+1}$ is of $H$-spaces and $H$-maps.

The next proposition and the following corollary give structural properties of the spaces $T, G$, and $R$.

Proposition 4.7 The spaces $T$ and $G$ are atomic.

Proof It is easy to see that $T$ is atomic using the product structure and the Bockstein relations. The case of $G$ is more difficult. We first show that $\alpha_{k}$ is essential for all $k$. Suppose not, so that for some $k$ we have a homotopy equivalence

$$
G_{k} \simeq G_{k-1} \vee P^{2 n p^{k}+1}\left(p^{r+k}\right) .
$$

Using the retraction $G_{k} \rightarrow G_{k-1}$ we construct a composition

$$
T^{2 n p^{k+1}-2} \stackrel{g_{k+1}}{\longrightarrow} \Omega G_{k} \rightarrow \Omega G_{k-1} \stackrel{h_{k-1}}{\longrightarrow} T
$$

which induces an isomorphism in $H^{2 n}$. Using the cup product and Bockstein structure, we infer that this composition is a cohomology isomorphism in dimensions less than $2 n p^{k+1}-1$. Since $H^{2 n p^{k}}(T) \simeq Z / p^{r+k}$, there must be an element of $H^{2 n p^{k}}\left(\Omega G_{k-1}\right)$ of order $p^{r+k}$. This contradicts Theorem 4.3 (i). 
Now suppose $\gamma: G \rightarrow G$ is a cellular map inducing and isomorphism in $H_{2 n}$. We will show, by induction on $k$, that $\gamma_{p}: H_{2 n p^{k}}(G) \rightarrow H_{2 n p^{k}}(G)$ is an isomorphism. Assuming that $\gamma_{*}$ is an isomorphism in degrees less that $2 n p^{k}$, consider the diagram

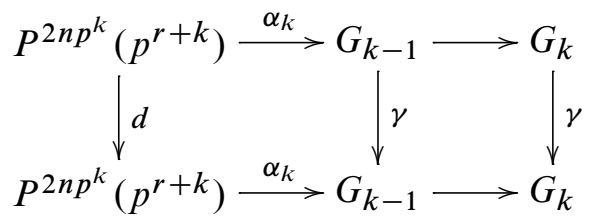

where $d$ is an integer multiple of the identity map. Since $d \alpha_{k}=\alpha_{k}$ and $\alpha_{k}$ is nontrivial, $d$ is a $p$ local unit and $\gamma_{*}$ is an isomorphism in dimension $2 n p^{k}$ by the 5 -lemma.

Corollary 4.8 $R \in \mathcal{W}_{r}^{\infty}$.

Proof Since $G$ and $T$ are atomic, Theorem 4.4 implies that $(G, T)$ is a corresponding pair in the sense of [11]. According to [11, Theorem 3.2], $R$ is a retract of $\Sigma T \wedge T$. However $\Sigma T \wedge T \in \mathcal{W}_{r}^{\infty}$ by Theorem 4.3(m).

The next proposition implies that the space $T$ constructed in this paper is homotopy equivalent to the space Anick constructed in [1] when $p \geq 5$ (the primes for which Anick's construction holds).

Proposition 4.9 Suppose $X$ is an $H$ space and there is a fibration sequence

$$
\Omega^{2} S^{2 n+1} \stackrel{\varphi}{\longrightarrow} S^{2 n-1} \stackrel{i}{\longrightarrow} X
$$

such that the composite

$$
\Omega^{2} S^{2 n+1} \stackrel{\varphi}{\longrightarrow} S^{2 n-1} \stackrel{E^{2}}{\longrightarrow} \Omega^{2} S^{2 n+1}
$$

is homotopic to the $p^{r}$ power map. Then $X \simeq T$.

Proof Consider the diagram of fibrations

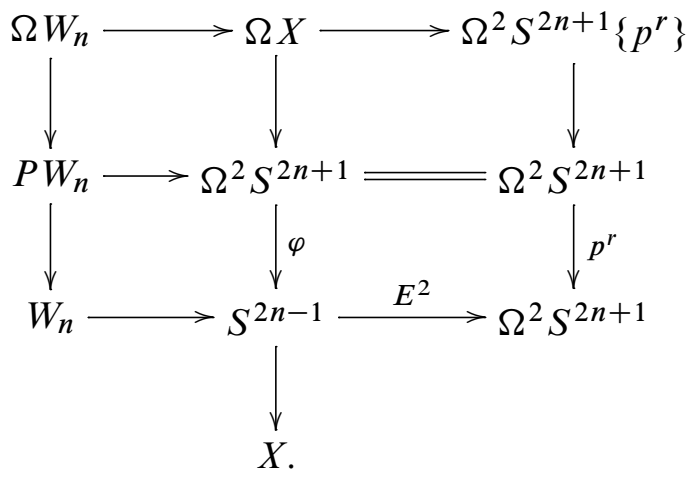


Since $p \cdot \pi_{*}\left(W_{n}\right)=0$ and $p^{r} \cdot \pi_{*}\left(S^{2 n+1}\left\{p^{r}\right\}\right)=0$ we conclude that $p^{r+1} \cdot \pi_{*}(X)=0$. Since $\pi_{2 n p-1}\left(W_{n}\right)=0$, we also see that $p^{r} \cdot \pi_{2 n p-1}(X)=0$. According to [2, Corollary 4.2] this is sufficient to construct a map

$$
\varphi: G \longrightarrow \Sigma X
$$

which induces an isomorphism in $\pi_{2 n}$. The construction given in [2] depends only on the co- $H$ space structure on $G$ and the fact that $\alpha_{k}$ is divisible by $p^{r+k-1}$, so the proof works in this context as well. From this we construct the composition

$$
T \stackrel{g}{\longrightarrow} \Omega G \stackrel{\Omega \varphi}{\longrightarrow} \Omega \Sigma X \longrightarrow X .
$$

It is an easy calculation with the Serre spectral sequence that $H^{*}(X ; \mathbb{Z} / p \mathbb{Z}) \cong$ $H^{*}(T ; \mathbb{Z} / p \mathbb{Z})$, so this map is a homotopy equivalence.

\section{References}

[1] D Anick, Differential algebras in topology, Research Notes in Math. 3, A K Peters Ltd., Wellesley, MA (1993) MR1213682

[2] D Anick, B Gray, Small H spaces related to Moore spaces, Topology 34 (1995) 859-881 MR1362790

[3] F R Cohen, The unstable decomposition of $\Omega^{2} \Sigma^{2} X$ and its applications, Math. Z. 182 (1983) 553-568 MR701370

[4] F R Cohen, J C Moore, J A Neisendorfer, Decompositions of loop spaces and applications to exponents, from: "Algebraic topology, Aarhus 1978 (Proc. Sympos.)", (J L Dupont, I H Madsen, editors), Lecture Notes in Math. 763, Springer, Berlin (1979) 1-12 MR561210

[5] F R Cohen, J C Moore, J A Neisendorfer, The double suspension and exponents of the homotopy groups of spheres, Ann. of Math. (2) 110 (1979) 549-565 MR554384

[6] F R Cohen, J C Moore, J A Neisendorfer, Torsion in homotopy groups, Ann. of Math. (2) 109 (1979) 121-168 MR519355

[7] B Gray, A note on the Hilton-Milnor theorem, Topology 10 (1971) 199-201 MR0281202

[8] B Gray, On the iterated suspension, Topology 27 (1988) 301-310 MR963632

[9] B Gray, EHP spectra and periodicity. I. Geometric constructions, Trans. Amer. Math. Soc. 340 (1993) 595-616 MR1152323

[10] B Gray, EHP spectra and periodicity. II. $\Lambda$-algebra models, Trans. Amer. Math. Soc. 340 (1993) 617-640 MR1152324 
[11] B Gray, On decompositions in homotopy theory, Trans. Amer. Math. Soc. 358 (2006) 3305-3328 MR2218977

[12] IM James, Reduced product spaces, Ann. of Math. (2) 62 (1955) 170-197 MR0073181

[13] M Mahowald, On the double suspension homomorphism, Trans. Amer. Math. Soc. 214 (1975) 169-178 MR0438333

[14] M Mather, Pull-backs in homotopy theory, Canad. J. Math. 28 (1976) 225-263 MR0402694

[15] J A Neisendorfer, 3-primary exponents, Math. Proc. Cambridge Philos. Soc. 90 (1981) 63-83 MR611286

[16] J A Neisendorfer, The exponent of a Moore space, from: "Algebraic topology and algebraic $K$-theory (Princeton, N.J., 1983)", (W Browder, editor), Ann. of Math. Stud. 113, Princeton Univ. Press (1987) 35-71 MR921472

[17] J Neisendorfer, Algebraic methods in unstable homotopy theory, New Math. Monogr. 12, Cambridge Univ. Press (2009)

[18] S D Theriault, A reconstruction of Anick's fibration, $\mathrm{PhD}$ thesis, University of Toronto (1997)

[19] SD Theriault, Properties of Anick's spaces, Trans. Amer. Math. Soc. 353 (2001) 1009-1037 MR1709780

[20] SD Theriault, The 3-primary classifying space of the fiber of the double suspension, to appear in Proc. Amer. Math. Soc.

Department of Math, Stats and Comp Sci, University of Illinois at Chicago 851 S Morgan Street, Chicago, IL, 60607-7045, USA

Department of Mathematical Sciences, University of Aberdeen Aberdeen AB24 3UE, United Kingdom brayton@uic.edu, s.theriault@maths.abdn.ac.uk

Proposed: Paul Goerss

Seconded: Bill Dwyer, Ralph Cohen
Received: 18 December 2007

Revised: 3 August 2009 